\title{
A Design Study of the Parallel-Slit Ring Collimator for Fast Neutron Emission Tomography of Spent Fuel
}

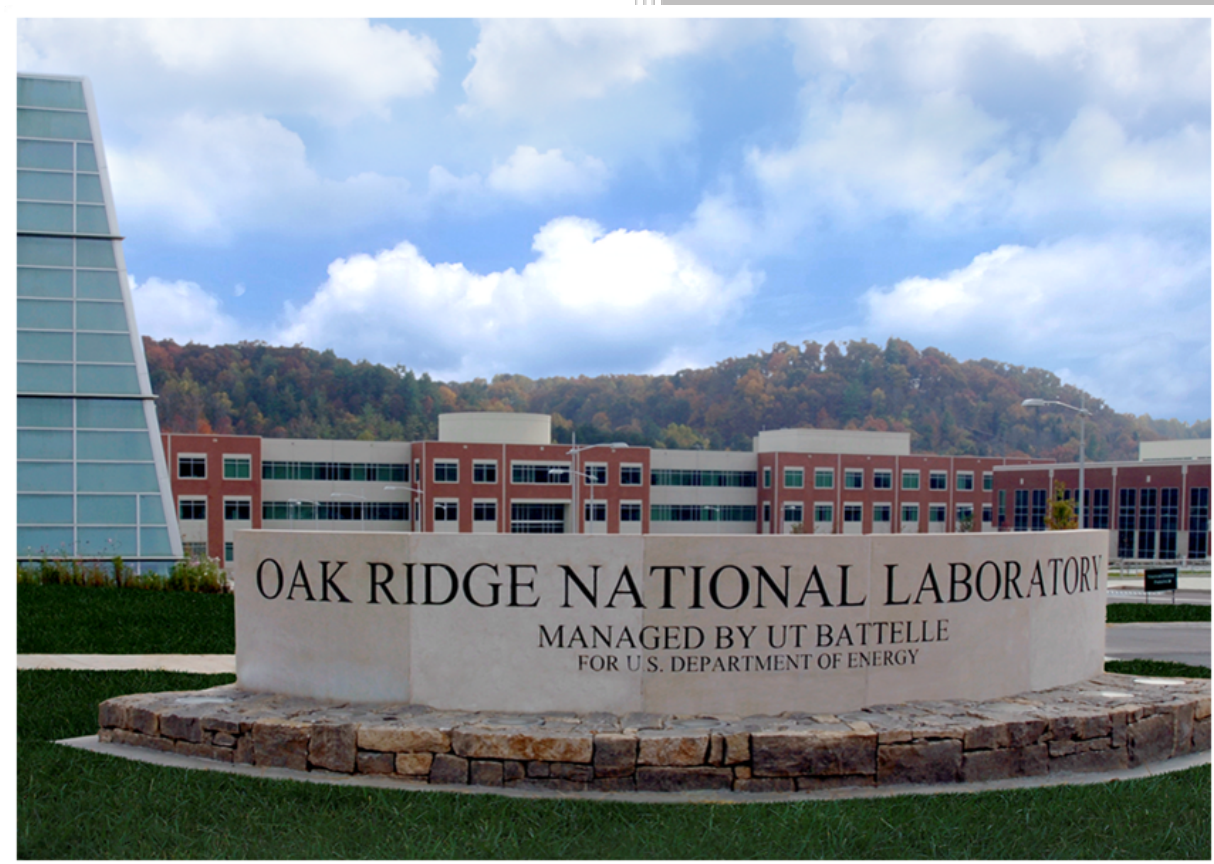

P. A. Hausladen

A. S. lyengar

L. Fabris

J. Yang

J. $\mathrm{Hu}$

September 2018 


\title{
DOCUMENT AVAILABILITY
}

Reports produced after January 1, 1996, are generally available free via US Department of Energy (DOE) SciTech Connect.

Website www.osti.gov

Reports produced before January 1, 1996, may be purchased by members of the public from the following source:

\author{
National Technical Information Service \\ 5285 Port Royal Road \\ Springfield, VA 22161 \\ Telephone 703-605-6000 (1-800-553-6847) \\ TDD 703-487-4639 \\ Fax 703-605-6900 \\ E-mail info@ntis.gov \\ Website http://classic.ntis.gov/
}

Reports are available to DOE employees, DOE contractors, Energy Technology Data Exchange representatives, and International Nuclear Information System representatives from the following source:

Office of Scientific and Technical Information

PO Box 62

Oak Ridge, TN 37831

Telephone 865-576-8401

Fax 865-576-5728

E-mail reports@osti.gov

Website http://www.osti.gov/contact.html

This report was prepared as an account of work sponsored by an agency of the United States Government. Neither the United States Government nor any agency thereof, nor any of their employees, makes any warranty, express or implied, or assumes any legal liability or responsibility for the accuracy, completeness, or usefulness of any information, apparatus, product, or process disclosed, or represents that its use would not infringe privately owned rights. Reference herein to any specific commercial product, process, or service by trade name, trademark, manufacturer, or otherwise, does not necessarily constitute or imply its endorsement, recommendation, or favoring by the United States Government or any agency thereof. The views and opinions of authors expressed herein do not necessarily state or reflect those of the United States Government or any agency thereof. 
Isotope and Fuel Cycle Technology Division

\title{
A DESIGN STUDY OF THE PARALLEL-SLIT RING COLLIMATOR FOR FAST NEUTRON EMISSION TOMOGRAPHY OF SPENT FUEL
}

\author{
P. A. Hausladen \\ A. S. Iyengar \\ L. Fabris \\ J. Yang \\ J. Hu
}

Date Published: September 2018

Prepared by

OAK RIDGE NATIONAL LABORATORY

Oak Ridge, TN 37831-6283

managed by

UT-BATTELLE, LLC

for the

US DEPARTMENT OF ENERGY

under contract DE-AC05-00OR22725 



\section{CONTENTS}

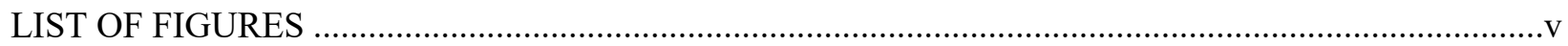

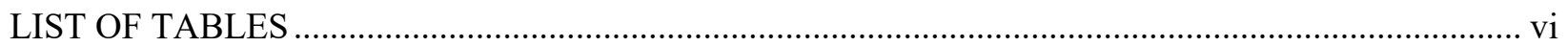

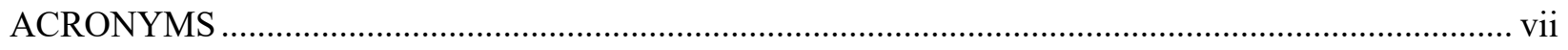

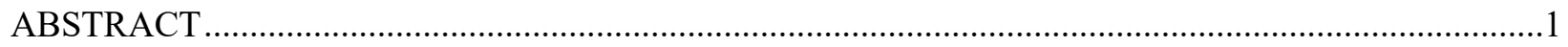

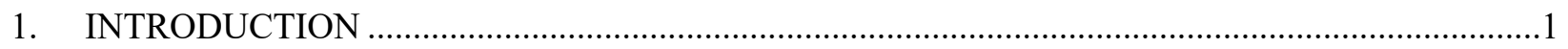

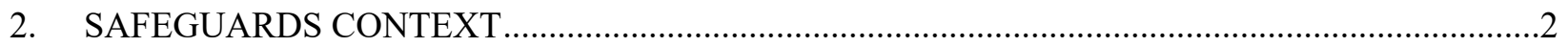

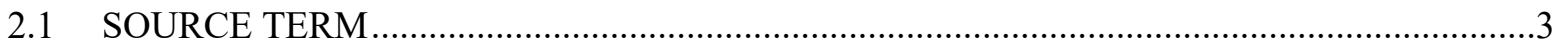

3. THE PARALLEL-SLIT RING COLLIMATOR ....................................................................

3.1 COLLIMATION AND LINES OF RESPONSE .........................................................

3.2 PARALLEL-SLIT RING COLLIMATOR CONCEPT .....................................................5

3.3 THE BASELINE IMAGER DESIGN ..................................................................

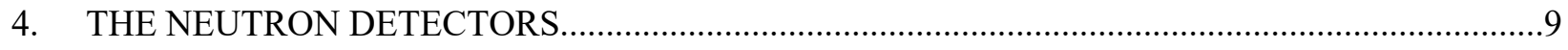

4.1 OPTIMAL STRAW CONFIGURATION ................................................................10

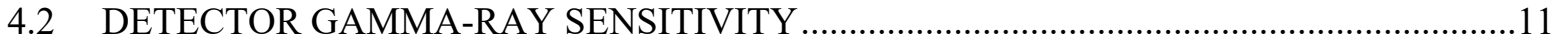

5. SIMULATION DESIGN STUDY ................................................................................

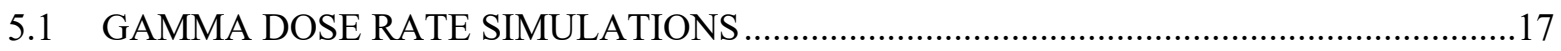

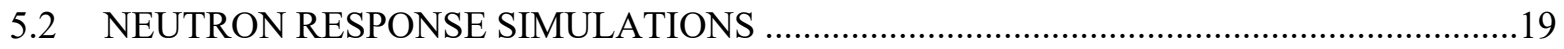

5.2.1 The "direct" and "edge" components of the response ............................................20

5.2.2 Inter-detector scattering ...........................................................................24

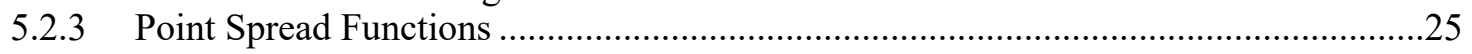

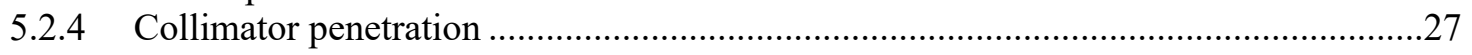

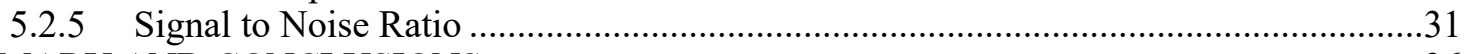

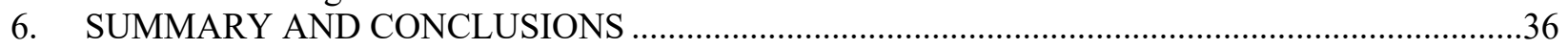

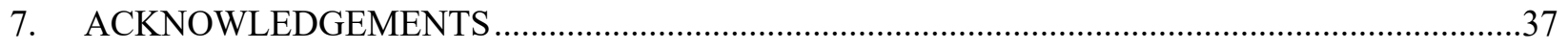

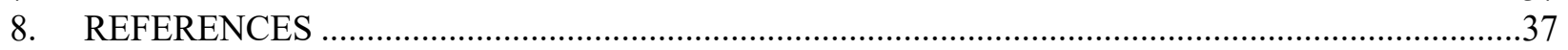





\section{LIST OF FIGURES}

Figure 1. (a) Plutonium-239 content as a function of fuel assembly burnup or exposure...........................4

Figure 2. Example lines of response for a detector counting neutrons that exit a single $3 \mathrm{~mm}$ wide collimator slit shown (a) as a schematic diagram. ..............................................................5

Figure 3. The parallel-slit ring collimator in (e) is equivalent to the parallel-slit collimator shown

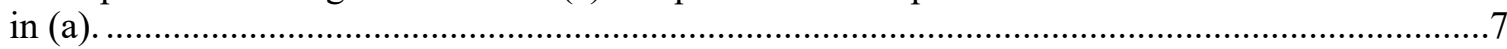

Figure 4. 2D view of the collimator: stainless steel (green), borated poly (yellow), and neutron

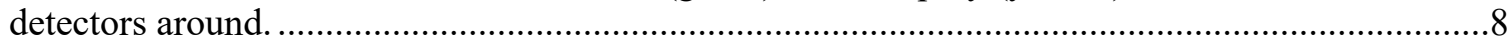

Figure 5. Three-dimensional configuration of the collimator and neutron detectors around a

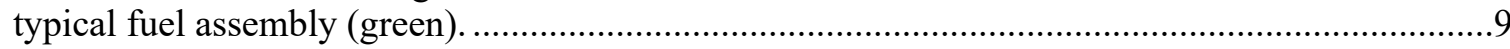

Figure 6. Various wedge and straw geometries. .................................................................................

Figure 7. Efficiency as a function of boron carbide coating thickness for the three different straw

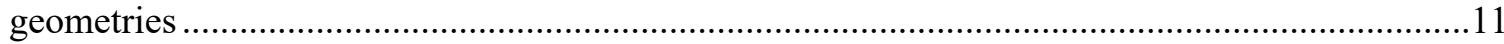

Figure 8. The prototype detector during irradiation experiments. .......................................................12

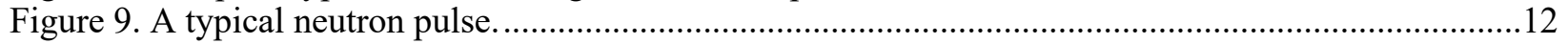

Figure 10. Oscilloscope traces corresponding to the four gamma dose rates to which the detector

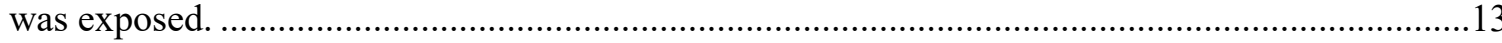

Figure 11. Pulse height spectrum vs. energy (MeV) from an MCNP simulation of (a) a beam of $662-\mathrm{keV}$ photons incident on a single detector and (b) the corresponding pulse height spectrum for neutrons.

Figure 12. Measured (solid) and simulated (dashed) counts per second in a single detector module as a function of detector threshold for different dose rate exposures.

Figure 13. Conceptual three-dimensional rendering of the baseline imager mounted in a GNS transport cask.

Figure 14. Map of gamma dose rates seen at all the detector straws for a collimator with $10 \mathrm{~cm}$ stainless steel, $30 \mathrm{~cm}$ borated polyethylene, and $3 \mathrm{~mm}$ parallel slits with a $40 \mathrm{GWd} / \mathrm{MTU}$ burnup fuel with 1-year cooling time as the source term.

Figure 15. Dose rates of the detectors with the (black) maximum and (red) minimum dose rate for each collimator configuration compared to the configuration average dose rate.

Figure 16. Maximum detector gamma dose rates per centimeter stainless steel for all 512 cases as a function of the collimator areal density...

Figure 17. The simplified collimator configuration with a single slit.

Figure 18. (Left) The response of the detector at the slit terminus to a neutron source at 285 different positions on a line perpendicular to the slit centerline and $20.16 \mathrm{~cm}$ from the slit opening, and (right) the same data displayed to accentuate the portion. .......................................22

Figure 19. Magnitude of the direct component of detector response as a function of slit width................22

Figure 20. Single slit geometry showing the projection of the slit width in the FOV.............................23

Figure 21. The scaled intensity for the sources at all three distances from the slit opening as a function of the source to slit edge distance in terms of the projected width W'.

Figure 22. (Above) schematic diagram of 11 detectors with a color scale representation of boron absorption reactions seen in each straw when a beam of ${ }^{244} \mathrm{Cm}$ neutrons is shot to the center detector only.

Figure 23. Diagram showing the areas overlapped by the fields of view of slits corresponding to detectors 50 and 51 .

Figure 24. Point spread functions for a ${ }^{244} \mathrm{Cm}$ line source at two different positions within the FOV of the baseline imager (a collimator with $10 \mathrm{~cm}$ stainless steel and $30 \mathrm{~cm}$ borated poly and $3 \mathrm{~mm}$ wide parallel slits).

Figure 25. Ratio of neutron current leaving the detectors to the neutron current reflected back into the detectors.

Figure 26. The effective thickness of the collimator inferred from the collimator penetration of the response as a function of the average thickness of borated polyethylene. 
Figure 27. The effective thickness of a solid collimator in mean free paths as a function of the thickness of borated polyethylene.

Figure 28. The difference between the calculated thickness (4) and the effective thickness of the collimator as a function of the thickness of borated polyethylene removed by the slits.

Figure 29. Estimated thickness, using Equation (4), as a function of the effective thickness of the collimator for configurations with $5 \mathrm{~cm}$ of stainless steel.

Figure 30. Image showing position of single pin and its associated halo.

Figure 31. (Left) the baseline imager with detector 50 indicated in red, (center) the response to detector 50, and (right) the response to detector 50 including inter-detector scattering.................33

Figure 32. SNR for all 512 cases as a function of the slit harmonic mean.

Figure 33. SNR as a function of the maximum gamma dose rate for each collimator geometry having a total collimator thickness less than or equal to $45 \mathrm{~cm}$. .34

Figure 34. The SNR and dose rate as a function of slit width for parallel and tapered slits .35

Figure 35. SNR as a function of the maximum gamma dose rate for collimator configurations consisting of $10 \mathrm{~cm}$ stainless steel and $30 \mathrm{~cm}$ of borated polyethylene

\section{LIST OF TABLES}

Table 1. Different combinations of stainless steel and borated polyethylene thicknesses being considered for the simulations going forward based on size constraints of two commercially available transport casks. 


\section{ACRONYMS}

CT computed tomography

DCVD Digital Cerenkov Viewing Device

FDET fork detector

FOV field of view

IAEA International Atomic Energy Agency

NDA nondestructive assay

ORNL Oak Ridge National Laboratory

PSF

PTI

PWR

SNR

point spread function

Proportional Technologies Inc.

pressurized water reactor

signal-to-noise ratio 



\begin{abstract}
For the past 18 months, Oak Ridge National Laboratory has been developing a passive fast-neutron emission tomography capability. The goal of this development is a new passive capability to detect diversion of single fuel pins (rods) from spent or used nuclear fuel assemblies by performing neutron emission tomography. At present, a laboratory prototype imager is under construction. The purpose of the prototype imager is to demonstrate imaging capability sufficient to resolve individual fuel pins in a spent nuclear fuel assembly. This report documents the results of simulation studies to optimize the design of the prototype imager.
\end{abstract}

\title{
1. INTRODUCTION
}

Oak Ridge National Laboratory (ORNL) is developing a new capability for passive fast-neutron emission tomography. Like the passive gamma emission tomography capability presently being evaluated by the International Atomic Energy Agency (IAEA), the goal of this passive neutron emission tomography capability is to detect the removal or substitution of individual fuel pins in spent nuclear fuel assemblies for international safeguards applications, such as verifying the integrity of an assembly before transfer to difficult-to-access storage. At present, a laboratory prototype imager is under construction. The purpose of the prototype imager is to demonstrate sufficient capability to resolve individual fuel pins in a spent nuclear fuel assembly. This report documents the results of simulation studies to optimize the design of the prototype imager and fulfills the deliverable "Physics Design of the Spent-fuel Imager" for the project "Detection of Fuel Pin Diversion via Fast Neutron Emission Tomography" supported by the Office of Defense Nuclear Nonproliferation Research and Development (NA-22).

Emission tomography works by using collimation to isolate activity along "lines of response" through an object, such as a fuel assembly. By combining many collimated views through the assembly, the neutron emission from each fuel pin can be mathematically extracted. Then, pin positions having reconstructed activity inconsistent with the appropriate rate of neutron emission can be identified. The use of fast neutrons to perform tomography is desirable because neutrons readily penetrate the high-atomic-number fuel in the assemblies, making the contribution of neutrons from fuel pins from the interior of the assembly readily measurable. However, the ability of fast neutrons to penetrate a good deal of shielding also makes them difficult to collimate and measure with the required resolution. Despite this difficulty, in previous work, ORNL demonstrated proof-of-concept emission-neutron computed tomography using fast fission neutrons that was capable of resolving individual pins of fresh plutonium mixed oxide fuel [1]. Unfortunately, this approach used high-resolution scintillation detectors that cannot withstand the overwhelming gamma-ray emissions from spent fuel.

To address the combined challenges associated with effectively collimating neutrons, achieving sufficient resolution, maintaining sufficient efficiency for the modest neutron source strength from spent fuel, and maintaining sufficient insensitivity to the gamma-ray flux to allow meaningful neutron measurements, a new collimator concept has been developed termed a "parallel-slit ring collimator." In this design, the slits of a parallel-slit collimator are rearranged to distribute them around a ring that surrounds the target fuel assembly. This rearrangement allows for the necessary increased separation of collimator slits and larger moderated neutron detectors. Moderated detectors offer the advantage of achieving their neutron efficiency in proportion to the volume of moderator, which is insensitive to gamma rays, and having comparatively little active volume that is sensitive to gamma rays. The prototype imager therefore comprises a parallel-slit ring collimator surrounded by a ring of commercially available boron straw detectors that can withstand the high gamma-ray dose rates from a typical spent fuel assembly.

The width of collimator slits and the thickness and materials of the collimator together determine the resolution, efficiency, and gamma-ray dose imparted to the detectors. The goal of the design optimization is to vary the collimator parameters to maximize the combination of resolution and efficiency to resolve 
individual fuel pins at a gamma dose rate that is tolerable to the detectors. A collimator comprised of an inner layer of steel and an outer layer of borated polyethylene combines to control gamma-ray dose and modulate neutrons. The simulations find that the resolution limitations imposed by inter-detector scattering favor wider slits as allowed by dose rate limitations. Furthermore, tapered slits that are narrower in the steel layer and wider in the borated polyethylene layer enable a combination of better gamma dose control and increased neutron detector efficiency.

This report opens with a description of the safeguards context for fast-neutron emission imaging. Then, the parallel-slit ring collimator is described, first conceptually, and then as part of the baseline imager design. Then, the neutron detectors and their response to high-dose gamma-ray fields are described to identify dose rates at which the detectors can operate. At this point, the simulated response to both gamma rays and neutrons of a suite of imager designs with different collimator thicknesses and slit widths is presented. Finally, using the response to neutrons, an estimated signal-to-noise ratio (SNR) is calculated for each configuration and the results shown as a function of gamma-ray dose rate and results presented.

\section{SAFEGUARDS CONTEXT}

Because of the thousands of metric tons of heavy metal being discharged from nuclear reactors globally, countries like Finland and Sweden are taking the lead to develop underground repositories to store their used nuclear fuel [2],[3]. The IAEA has developed safeguards approaches under integrated safeguards for encapsulation plants and geological repositories [4]. Spent fuel safeguards rely primarily on material containment and surveillance techniques along with item counting and nondestructive assay (NDA) verification measurements [5]. These types of measurements are required before spent fuel assemblies are transferred to long-term dry storage, final disposal at a repository, or other facilities where they are not easily accessible [3].

The majority of plutonium safeguarded by the IAEA is contained in spent nuclear fuel from light water reactors (LWRs). The LWR fuel assemblies are safeguarded as items, and absence of diversion is only confirmed when all items are accounted for after their integrity is verified. All nuclear material in LWR fuel assemblies used to remain in the same items during the whole irradiation lifetime of the fuel assemblies. However, since the 1980s, most LWR operators can replace leaking rods during planned refueling outages and as a result, a small population of assemblies has rods stripped out or replaced [6]. Several attempts have been made by the IAEA and its Member States to develop technologies to detect diversions of pins from a spent fuel assembly and to determine whether pins have gone missing or have been replaced with dummy or fresh fuel pins. This mode of verification is known as partial defect detection [2]. The IAEA partial defect test addresses a diversion scenario where irradiated pins are extracted from the fuel assembly or the pins are replaced with un-irradiated material, and no subsequent irradiation after the fuel rod substitution is envisaged. The current policy of the IAEA is to carry out a partial defect test on all easily dismountable spent fuel that is being transitioned to storage where reverification would be impossible or difficult, such as in dry storage casks, or repositories [5]. Although notionally a small fraction, a single pin extracted from each fuel assembly in the core of a single LWR would yield as much as $2.4 \mathrm{~kg}$ of ${ }^{239} \mathrm{Pu}$. Detection of this level of partial defect is approximately 2 orders of magnitude more sensitive than equipment currently employed by the IAEA.

The unit of special nuclear material in a reactor is a nuclear fuel assembly made up of an array of fuel rods (also called pins). For example, a four-loop pressurized water reactor (PWR) Westinghouse reactor core has 193 fuel assemblies, a third of which is replaced approximately every 12-18 months depending on the reactor design and operating history [7]. The instruments currently approved and in use for the partial defect testing of spent fuel for verification before transfer to dry storage are the fork detector (FDET) and the Digital Cerenkov Viewing Device (DCVD) [8], [9]. For these devices, the performance criteria for verifying the integrity of a spent fuel assembly means detecting a diversion of $50 \%$ of the material in an assembly with high confidence (90\%) [6]. The DCVD is less accurate in measuring fuel 
with long cooling times, low burnup, or in dirty pool conditions [2]. The DCVD also cannot detect a single replaced fuel rod and is limited in scenarios in which random pins are missing because adjacent fuel pins can cause the device to register a false negative [2], [8]. FDET measurements use both the passive neutron and gamma emissions from the spent fuel assembly to characterize it and cannot be employed to independently verify pin diversion [9]. FDET relies upon the facility operator's declared data to conduct verification of spent fuel assemblies before the fuel is transferred to dry storage casks [8]. According to the IAEA, a major weakness of both the DCVD and FDET is that the detection probability is insignificant for carefully designed low-level diversions of a few fuel rods in each fuel assembly within a large population [6].

The IAEA in conjunction with some Member State support programs is presently supporting the development of a passive gamma emission tomography system for partial and bias defect detection in spent fuel [8], [9]. This technique has been demonstrated to be capable of resolving individual fuel pins in fuel assemblies. It is envisioned that fast-neutron emission tomography could be used in similar circumstances. The output of the tomographic measurement and image reconstruction is the measured neutron emission rate of each fuel pin; this pin-by-pin accounting provides a robust check for the completeness of a fuel assembly against pin removals or substitution before transfer to difficult-to-access storage. However, neutron-based tomography measurements have a number of potential advantages:

- Neutron measurements may be sensitive to assemblies containing fuel pins that were replaced after a single cycle in the reactor and subsequently irradiated in the core. This sensitivity is a consequence of the neutron signal originating primarily from the ingrowth of ${ }^{244} \mathrm{Cm}$, which occurs predominantly at the end of an exposure cycle.

- Neutron measurements may have better sensitivity for resolving individual pins toward the center of larger fuel assemblies where the sensitivity of gamma emission tomography is limited by selfattenuation of the fuel assembly.

- Neutron measurements may be able to determine the fissile loading of each pin. In water, neutron leakage from a fuel assembly can be approximately three times the value in air; these additional neutrons originate from thermal neutron-induced fission of fissile isotopes. Measuring a fuel assembly in water and in air may enable the induced fission component to be isolated.

The neutron emission tomography technique is not specific to fuel types from LWRs and can be also be applied to fuels from reactors with greater proliferation risk, such as CANDU and fast reactors. Emission tomography methods could also be applied to fresh plutonium mixed oxide fuel, in which case they would measure the ${ }^{240} \mathrm{Pu}$-effective on a pin-by-pin basis.

\subsection{SOURCE TERM}

The goal of this project is to design and optimize an imaging system to verify the completeness of spent fuel assemblies using fast neutrons. Traditional methods of measuring neutrons from spent fuel result in the measurement of ${ }^{239} \mathrm{Pu}$ content. However, $\mathrm{Pu}$ content is only known to a few percent in burnup codes, and the effort to resolve any discrepancies in the operator declaration would prove too expensive and cumbersome for a safeguards inspectorate. Also, as can be seen in Figure 1(a), the Pu content saturates with increased burnup or exposure. This means that instruments that use this technique for spent fuel accountancy would be minimally sensitive to rod replacements made after the first cycle in a reactor. The neutron emission from spent nuclear fuel is dominated by the spontaneous fission of ${ }^{244} \mathrm{Cm}$, which has a half-life of 18.2 years and a neutron production rate of $1.64 \times 10^{7}$ neutrons s $\mathrm{gram}^{-1}$ [11]. A plot of the neutron rate (per meter per fuel rod) from spontaneous fission of ${ }^{244} \mathrm{Cm}$ in spent fuel having $3 \%$ initial enrichment, 2 years of cooling, and the prescribed exposure is shown in Figure 1(b) [10]. Note that for most commercial fuels the source strength per meter of fuel rod will exceed $10^{5} \mathrm{n} / \mathrm{s}$. Note also that this 
emission rate is many orders of magnitude lower than the gamma-ray emission rate. Unlike the $\mathrm{Pu}$ content, ${ }^{244} \mathrm{Cm}$ does not saturate as a function of burnup, so using this as the signature of choice would enable an imager to be sensitive to rod replacements made after the first cycle. For the initial simulations of the neutron response and point spread functions presented in this paper, a line source of ${ }^{244} \mathrm{Cm}$ was used.
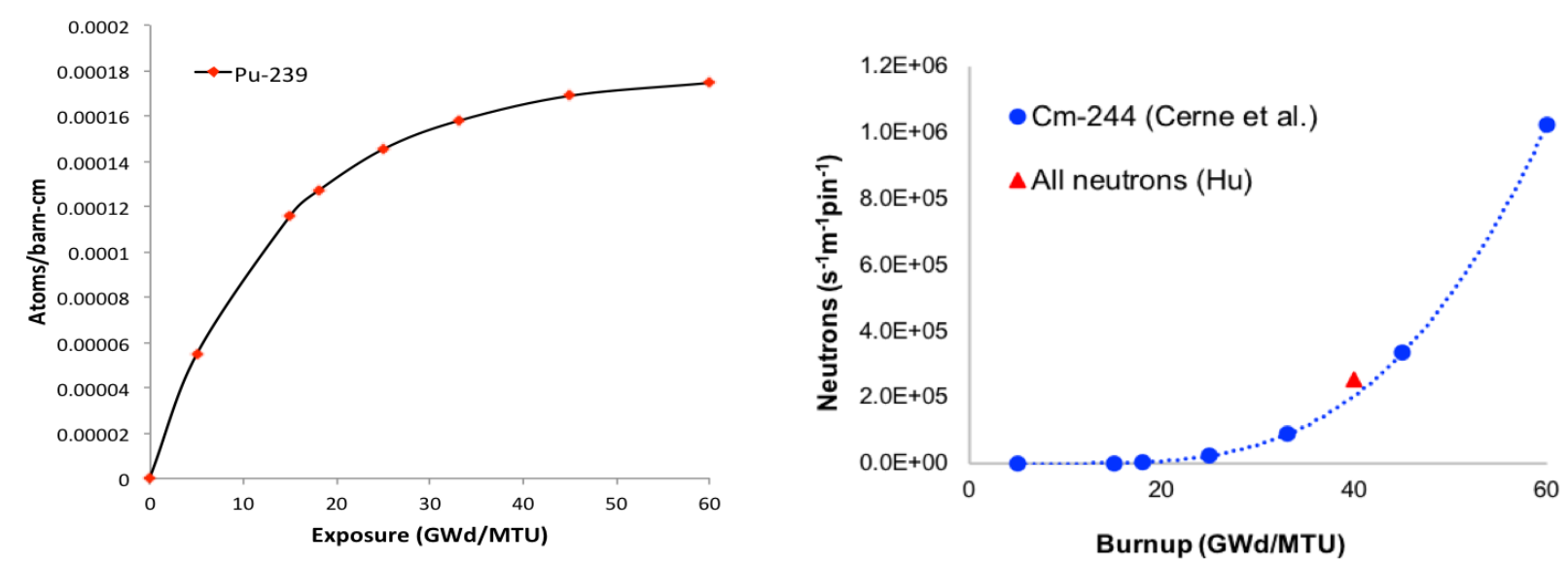

Figure 1. (a) Plutonium-239 content as a function of fuel assembly burnup or exposure. (b) Neutron rate per fuel rod due to ${ }^{244} \mathrm{Cm}$ as a function of burnup or exposure within an average commercial PWR (shown for $3 \%$ initial enrichment and 2 year cooling time) [10].

\section{THE PARALLEL-SLIT RING COLLIMATOR}

This section opens by describing the isolation of lines of response based on collimation. Then, the concept of the parallel-slit ring collimator is introduced. Finally, the baseline imager design is presented as a basis for comparison of a suite of imager designs with different dimensions.

\subsection{COLLIMATION AND LINES OF RESPONSE}

Most readers are familiar with the notion of computed tomography (CT) from medical physics, where xray $\mathrm{CT}$ has been commonplace for decades and emission tomography, such as positron emission tomography (PET) and single photon emission computed tomography (SPECT), also sees wide diagnostic use. Nonetheless, it is valuable to review aspects of CT that relate to design principles of the fast-neutron imager.

In each form of $\mathrm{CT}$, projection data from many angles are used to reconstruct cross-sectional images of an inspected object for diagnostic purposes. The crucial elements of this process are that measured data divide the object into lines of response, and multiple views through the object are mathematically combined to estimate an image. Here, a line of response refers to a path through the inspected object along which an observable (such as neutron emission activity) can be integrated. For passive neutron imaging, collimation is used to isolate such paths, or lines of response, through the fuel assembly, and neutron counts in a detector at the terminus of a collimator slit correspond primarily to an integral of neutron activity along the corresponding path. It is possible to achieve fast-neutron tomography with the desired resolution provided each line of response sufficiently isolates a path through the object, the object is sampled with a sufficient number of lines of response, and there are a sufficient number of views through the object to invert measured data to form an image.

To illustrate the concept of a line of response, a series of simulations were performed using a collimator consisting of an annulus of material with a single $3 \mathrm{~mm}$ wide slit cut in it. A schematic diagram of this geometry is shown in Figure 2(a). MCNP6 simulations were performed with a ${ }^{244} \mathrm{Cm}$ point source placed 
at the $17^{2}$ locations corresponding to a grid separated by the pin pitch of a $17 \times 17 \mathrm{PWR}$ fuel assembly. (However, note that no assembly was present in the simulations.) For each simulation, the neutron counts were tallied at the exit of the slit via the FMESH card with the F4 tally for calculating neutron flux through a cell. The resulting image in Figure 2(b) shows the neutron counts associated with each source location for a collimator thickness of $15 \mathrm{~cm}$. In this image, there is an identifiable path through the inspection volume that contributes more counts, but significant contributions remain from all the source positions. Similarly, Figure 2(c) shows the counts associated with each source location for a collimator thickness of $30 \mathrm{~cm}$. Here, almost all response is limited to a particular path across the inspection volume, but the larger collimator thickness reduces the total intensity.
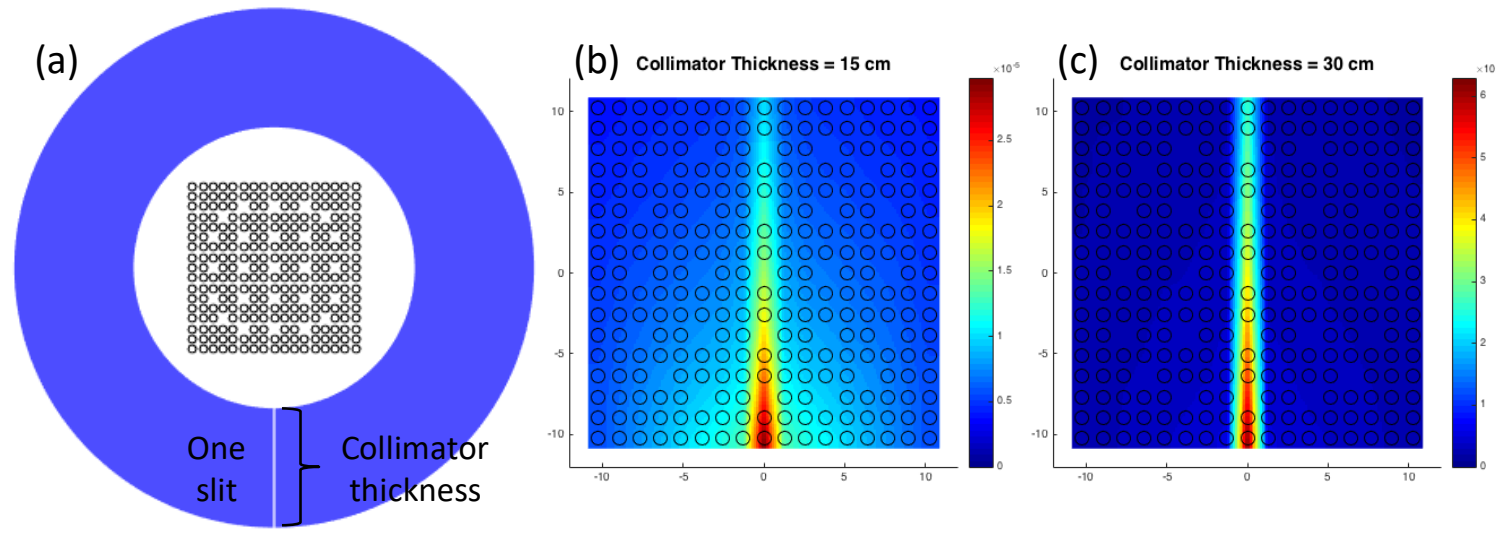

Figure 2. Example lines of response for a detector counting neutrons that exit a single $3 \mathbf{~ m m}$ wide collimator slit shown (a) as a schematic diagram. The results of a ${ }^{244} \mathrm{Cm}$ point source simulated at each assumed fuel pin location are shown (b) for a $15 \mathrm{~cm}$ thick collimator and (c) for a $30 \mathrm{~cm}$ thick collimator.

A parallel-slit collimator consists of a number of individual slits parallel to each other and defining lines of response across the inspection volume.

\subsection{PARALLEL-SLIT RING COLLIMATOR CONCEPT}

As indicated in the introduction, measuring fast neutrons is desirable because neutrons penetrate a good deal of shielding and readily escape from a fuel assembly. Unfortunately, their ability to penetrate shielding makes fast neutrons difficult to collimate and measure with fine spatial resolution. In addition, measuring the modest neutron source strength from spent fuel among the overwhelming gamma-ray emissions poses additional challenges. The challenges of using fast neutrons manifest themselves such that attributes of a detector or collimator that maximize one desirable characteristic (such as resolution) conflict with other desirable characteristics (such as efficiency). The intent of the present work is to make a functional equivalent to a parallel-slit collimator that can be used for large detector pixels in close proximity to the fuel. To do so, it is necessary to devise a design that simultaneously addresses the following challenges:

- Efficiency: The modest neutron source strength of spent fuel requires efficient use of the available neutrons, such as by placing large detectors close to the fuel and using wide collimator slits.

However, the modest neutron source strength also suggests that a sparse collimator design (implied by large detectors) combined with scanning would take too long, but removing collimator material to add slits or make them wider reduces the effectiveness of the remaining slits.

- Resolution: The need for resolution sufficient to resolve fuel pins can be restated as resolution sufficient to resolve the gap between fuel pins. Defining a line of response similar in size to the gap between fuel pins requires a slit width comparable to the gap dimension and a thick collimator from a 
good neutron moderator (like borated polyethylene). However, good neutron moderators do little to reduce gamma dose.

- Gamma-ray dose management: Managing the gamma-ray dose rate requires a combination of gamma blind detectors and a collimator that effectively shields gamma rays. The requirement for gammablind neutron detectors points toward moderated detectors where most of the energy loss is in moderator rather than in active detector volume. Moderated detectors, in turn, imply large detectors. Gamma dose rate can be reduced with denser collimator materials, such as steel or lead, but these materials are poor neutron moderators.

- Sufficient sampling: To reconstruct with resolution sufficient to identify individual fuel pins, it is desirable to sample the space between fuel pins. To do so for a conventional parallel-slit collimator requires a total of about 100 slits spaced across $35 \mathrm{~cm}$. It is recognized that a conventional parallel-slit collimator (due to the required detector element size or the efficiency of the collimator) can only work by reducing the number of slits and scanning the apparatus to achieve the required resolution. Although this solution is acceptable for the more numerous gamma rays, an increase of an order of magnitude (or more) in measurement time due to the necessity to scan would be unacceptable for neutrons.

- Compact overall size: For a number of detectors of specified size, a ring is the most compact geometry.

Despite these challenges, the use of a novel collimator concept appears to make it possible to construct an imager having sufficient resolution to identify individual fuel pins while also keeping the imager sufficiently compact, efficient, and radiation resistant to be practical. We call this novel collimator a "parallel-slit ring collimator." An imager using this collimator concept can use large detectors that are relatively close to the fuel with widely spaced collimator slits that simultaneously sample the entire volume.

This design is based on parallel slits but achieves the required spacing between slits (and detector elements) by rotating the position of each slit through a known angle to achieve equal detector spacing around the outside of the collimator annulus. In this way, the essential function of the collimator is maintained and correspondence to the parallel-slit collimator is maintained (i.e., isolating lines of response along particular chords across the central volume of the annulus). To illustrate, imagine a parallel-slit collimator that is part of an annulus of shielding. In the example shown in Figure 3(a), 20 slits are shown for ease of viewing. These slits are spaced too closely to modulate neutrons effectively, and in addition, this geometry would require small (millimeter scale) neutron detectors. Instead, the detectors can be moved farther apart by rotating each slit through a known angle to place the detectors at equiangular points around the outside of the collimator circle, as shown in Figure 3(e). Now, each slit creates the same line of response as it originally did but for a different projection angle (rotation of the object). For tomography, all projection angles (spaced around $360^{\circ}$ ) are measured, so an equivalent set of views will be acquired for the rotated slits as for the original parallel slits. An advantage of this collimator design is that the fuel and detectors can remain stationary, and only the collimator need rotate. As a result, fuel-detector positioning does not have to be made very precisely provided there is no relative motion during the measurement. 

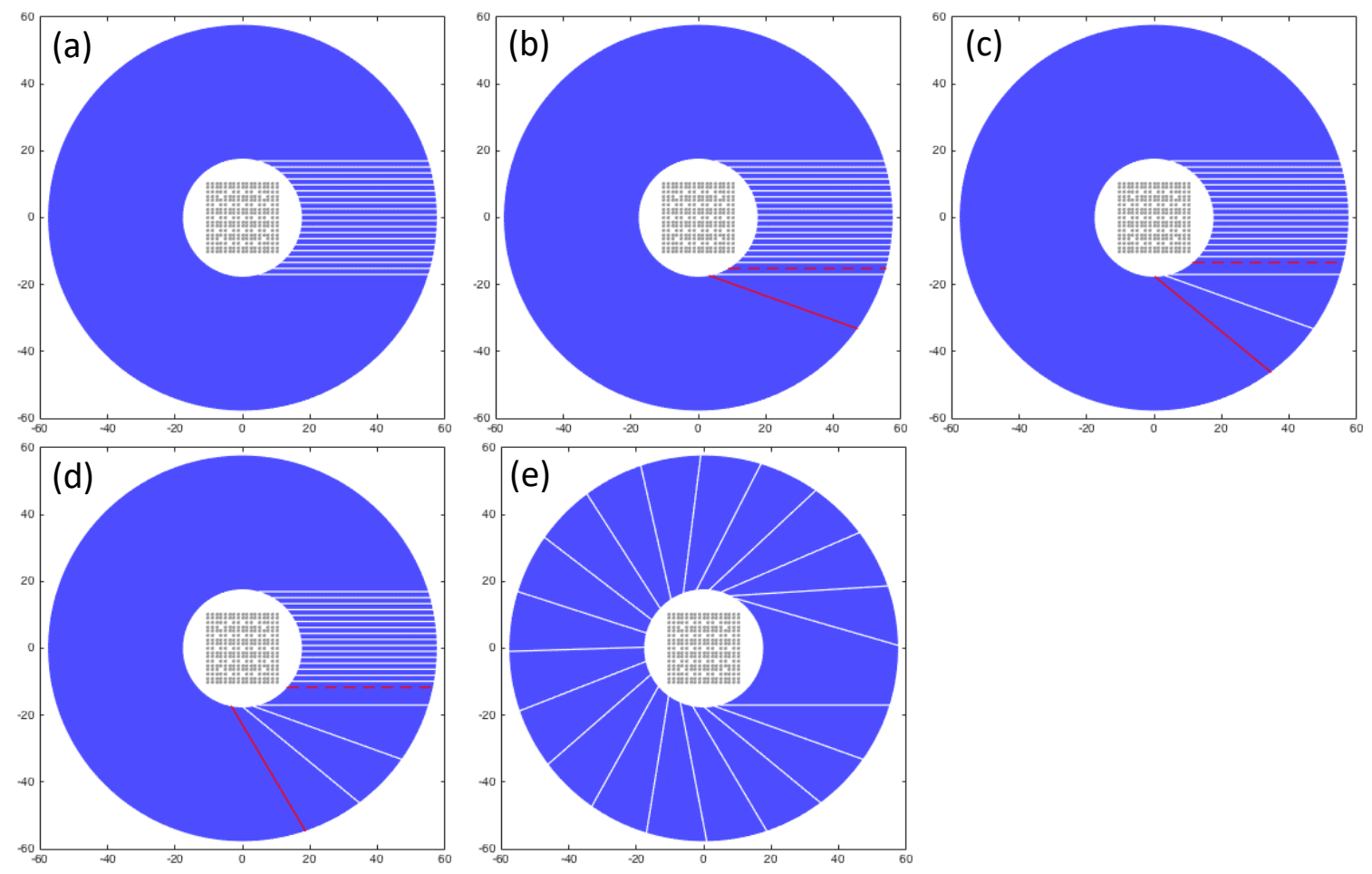

Figure 3. The parallel-slit ring collimator in (e) is equivalent to the parallel-slit collimator shown in (a). To get this collimator, as shown in (b)-(d), each slit is rotated from the dashed red to the solid red line so that after rotation, the resulting slits are (e) spaced equally around the circle. Each slit still inspects the same chord of the inspection volume.

\subsection{THE BASELINE IMAGER DESIGN}

It is useful to begin describing the imager by articulating a baseline design and the rationale for choosing particular dimensions or other parameters. Then, the parameters of the design can be studied to ascertain the effect on performance. Introducing a baseline design also provides an opportunity to describe the prototype imager more fully.

As discussed earlier, the imager consists of an annular collimator surrounded by a ring of detectors, where a detector is positioned at the outside end of each slit. Outside the detectors, there is an additional ring of shielding to reduce the background rate of neutrons in the detectors from potential surrounding sources. The inner diameter of the annulus is intended to accommodate most common LWR fuel assemblies, so it was chosen to accommodate assembly widths up to $24 \mathrm{~cm}$. In particular, the inner diameter of $17.67 \mathrm{~cm}$ was chosen to make its inscribed square $1 \mathrm{~cm}$ greater in side length than an assembly having a width of $24 \mathrm{~cm}$. The outer diameter of the imager was chosen so that it can be readily handled by a facility. By this, we mean that the imager can fit in a cask or cask-like container that operators typically handle. For the baseline imager, the outer diameter was chosen to fit within the internal cavity of the GNS Castor series of transport casks, which have an internal cavity diameter of $148 \mathrm{~cm}$, as this cask type is commonly used for international fuel shipments. (Note that there are casks with larger internal cavities, such as the Energy Solutions TS125 cask, which has an internal cavity diameter of $170 \mathrm{~cm}$.)

The number of slits in the collimator (and therefore the number of detectors) was chosen such that there are sufficient lines of response to sample the gaps between fuel pins. Common $17 \times 17$ fuel assemblies have inter-pin gaps of $0.328 \mathrm{~cm}$. Sampling $96 \%$ of the inner diameter of the collimator at a spacing equaling the inter-pin gap requires approximately 100 slits. As a result, the baseline imager design has 100 slits. 
A plan view of the baseline design is shown in Figure 4. The innermost $10 \mathrm{~cm}$ of the collimator is constructed of stainless steel (green), providing both gamma-ray shielding and structural integrity. Surrounding the stainless steel, there is a further $35 \mathrm{~cm}$ of collimator constructed from borated polyethylene (yellow). Collimation is achieved by one hundred $3 \mathrm{~mm}$ wide slits in the collimator, each terminating on a detector (gray).
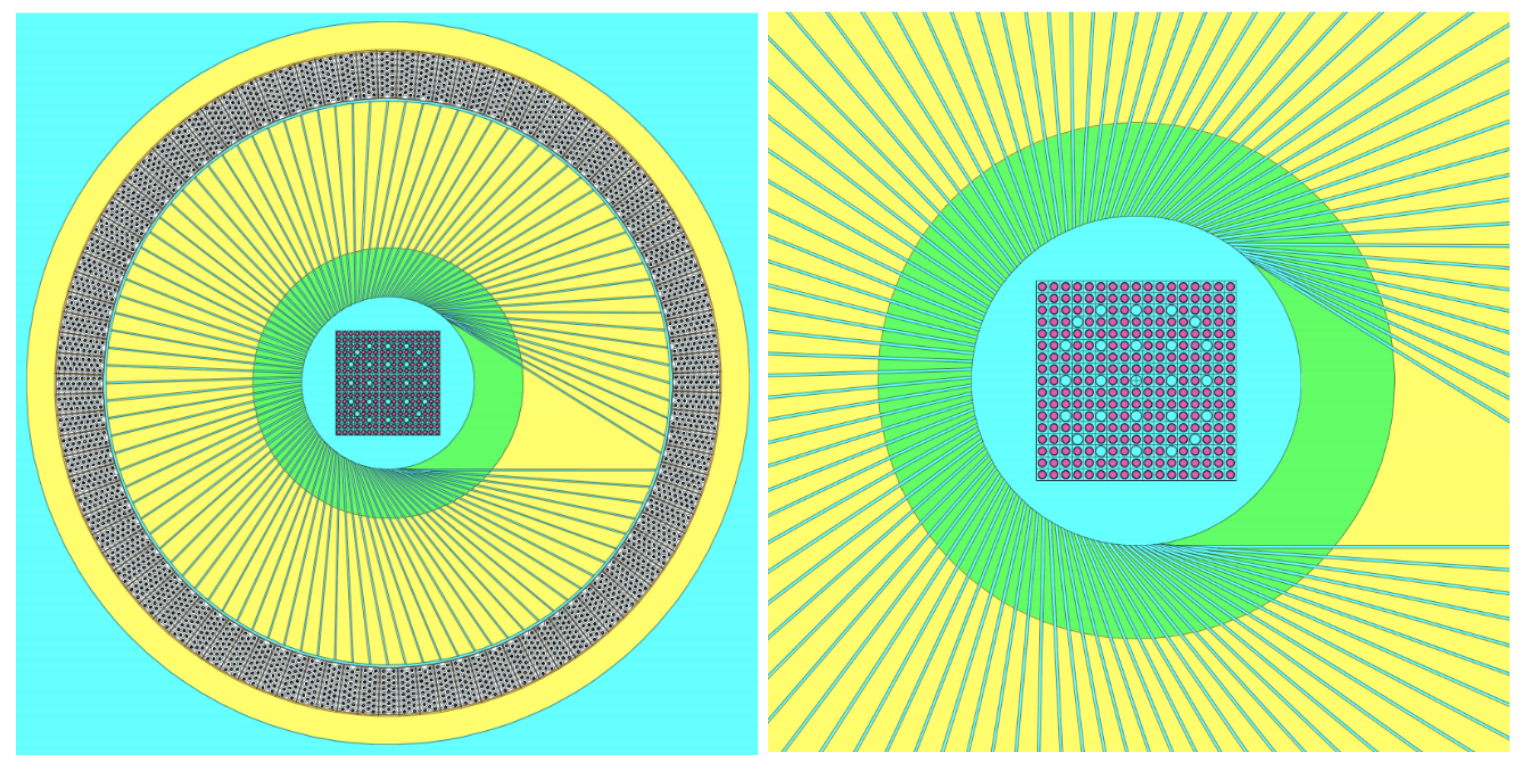

Figure 4. 2D view of the collimator: stainless steel (green), borated poly (yellow), and neutron detectors around.

The $3 \mathrm{~mm}$ slit width was chosen to be approximately the same as the slit spacing, so additional slit width will sample some of the same activity as neighboring slits. Each detector is wedge-shaped in cross section, has an active length of $1 \mathrm{~m}$, and is composed of 23 boron straws embedded in high-density polyethylene moderator. Note that the detectors will be described more fully in the next section. The active length was chosen to be as large as possible to maximize efficiency without compromising resolution. The neutron count rate scales like the square of the detector height; one factor of height comes from the larger source strength viewed by a longer detector, and one factor of height comes from the greater efficiency of the longer detector. However, the detector length is limited by the need to minimize blur in images due to measuring a single cross-sectional image for the entire length of fuel measured by the imager. After irradiation in the reactor core, fuel assemblies may warp by as much as $1 \mathrm{~cm}$ over their lengths. The height of the imager is limited such that the amount of warp encountered over the length of fuel in the imager is smaller than the inter-pin distance. A $1 \mathrm{~m}$ section of fuel should have a maximum displacement from top to bottom of $0.25 \mathrm{~cm}$, smaller than the inter-pin spacing. A three-dimensional rendering of the baseline configuration of the collimator and neutron detectors around a typical fuel assembly is shown in Figure 5. 

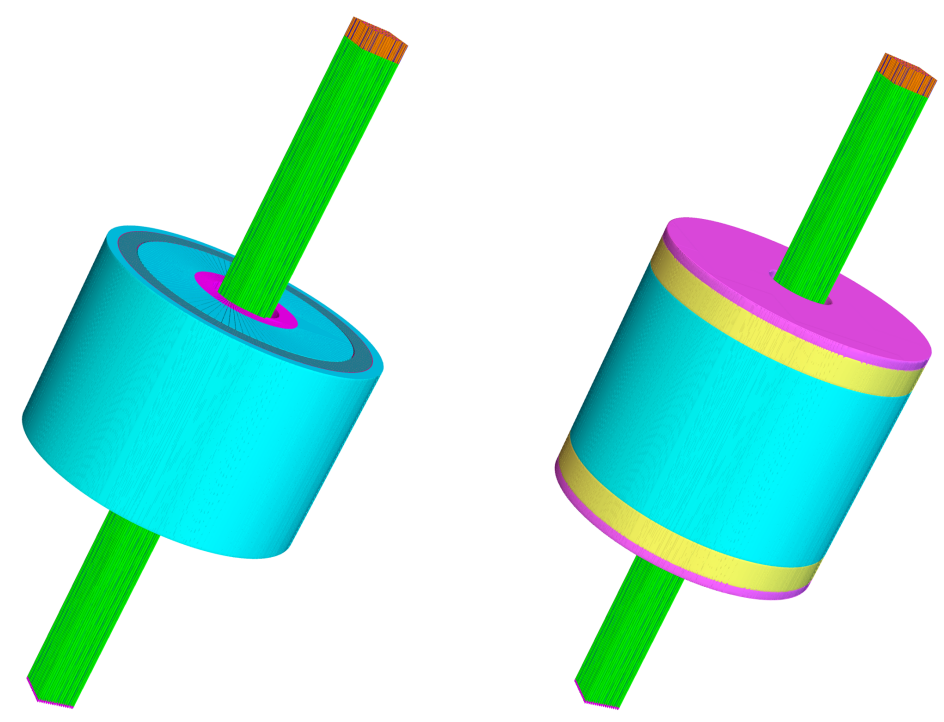

Figure 5. Three-dimensional configuration of the collimator and neutron detectors around a typical fuel assembly (green). The imager is shown (left) without and (right) with stainless steel (pink) and borated polyethylene shielding (yellow) at the top and bottom of the imager to shield from the fuel that extends above and below the imager.

Once a baseline design has been determined, aspects of the design can be varied to further optimize it. For instance, the collimator thickness, slit width, slit design (parallel vs. tapered), and fraction of the collimator consisting of borated poly and stainless steel each contribute to the performance of a particular design.

To optimize the design, better-performing designs need to be identified. With that in mind, in the remainder of this report, the ability of detectors to withstand gamma dose will be quantified. Then, the performance of different designs for controlling gamma dose will be assessed. Last of all, the correspondence of different designs to expected neutron SNR in neutron emission images needs to be established.

\section{THE NEUTRON DETECTORS}

Due to their inherent properties and the shielding provided by the collimator, the neutron detectors used in the present work deserve additional discussion because they need to be sufficiently gamma blind so that they can operate in close $(50-60 \mathrm{~cm})$ proximity to the spent fuel. For this application, boron-straw detectors were selected because of their combination of gamma blindness, efficiency, and cost per volume instrumented.

For the boron straw detectors, each detector consists of a number of boron straws embedded in high-density polyethylene moderator. Each straw is an approximately $4 \mathrm{~mm}$ diameter gas-filled aluminum tube that contains a ${ }^{10} \mathrm{~B}_{4} \mathrm{C}$-coated copper straw at the outer diameter and an anode wire in the center. Neutrons are detected via the neutron capture reaction ${ }^{10} \mathrm{~B}+n \rightarrow{ }^{7} \mathrm{Li}+\alpha$ reaction when either the $0.84 \mathrm{MeV}{ }^{7} \mathrm{Li}$ or $1.47 \mathrm{MeV} \alpha$ escapes the ${ }^{10} \mathrm{~B}_{4} \mathrm{C}$ coating and creates sufficient ionization in the fill gas of the straw to exceed the detector threshold. The straws are resilient to high gamma-ray fields because (1) most of the detector consists of inert moderator, (2) the small gas volume per straw and low gas pressure minimize the amount of material where gamma rays (and scattered electrons) can interact, and (3) the small radius of the detector means that the signals are inherently fast (tens of nanoseconds rather than microseconds) to minimize the amount of gamma-ray pileup. The ${ }^{10} \mathrm{~B}$ straws are manufactured by Proportional Technologies Inc. (PTI). Although the detectors detect neutrons at thermal energies, by incorporating boron in the collimator and 
placing a cadmium thermal-neutron filter between the collimator and detector, the imager still responds primarily to fast neutrons.

\subsection{OPTIMAL STRAW CONFIGURATION}

For this application, it was desirable to maximize neutron efficiency while minimizing gamma-ray efficiency. In practice, minimizing gamma-ray efficiency corresponds to minimizing the number of boron straw detectors per readout channel, thereby minimizing gamma-ray pileup. To investigate the most favorable configurations of boron straws, the efficiency was calculated as a function of the thickness of the ${ }^{10} \mathrm{~B}_{4} \mathrm{C}$ coating and the spacing of straws in a high-density polyethylene matrix. When calculating the efficiency of the boron straws, the overall detection efficiency is the product of the absorption fraction, the wall-escape efficiency, and the threshold efficiency. The absorption fraction is obtained from simulation (MCNP). The wall escape efficiency is the fraction of reaction products that escape the ${ }^{10} \mathrm{~B}_{4} \mathrm{C}$ coating, and enter the gas, where they can be counted. This number is a function of the ${ }^{10} \mathrm{~B}_{4} \mathrm{C}$ coating thickness, $T$, (in micrometers). The threshold efficiency is the fraction of events counted above the discriminator threshold. For most straw-detector applications, the fraction of pulse heights above threshold is about 0.95 . However, for high dose rate environments, the threshold efficiency can be significantly lower.

Information regarding the expected efficiency of the straw detectors as a function of the straw pitch and boron carbide coating thickness was supplied by the manufacturer. A schematic diagram of the different straw pitches that span typical use is shown in Figure 6. Likewise, the results from the efficiency simulations is shown in Figure 7. Note that although a straw pitch of $1.1 \mathrm{~cm}$ and a coating thickness of $1.2 \mu \mathrm{m}$ delivers the highest overall efficiency, the option with a straw pitch of $1.2 \mathrm{~cm}$ and a coating thickness of $1.4 \mu \mathrm{m}$ and yields $97.5 \%$ the efficiency of the most efficient configuration while using the fewest number of straws ( 23 straws per detector for a total of 2,300 straws), making it more economical and less gamma sensitive.
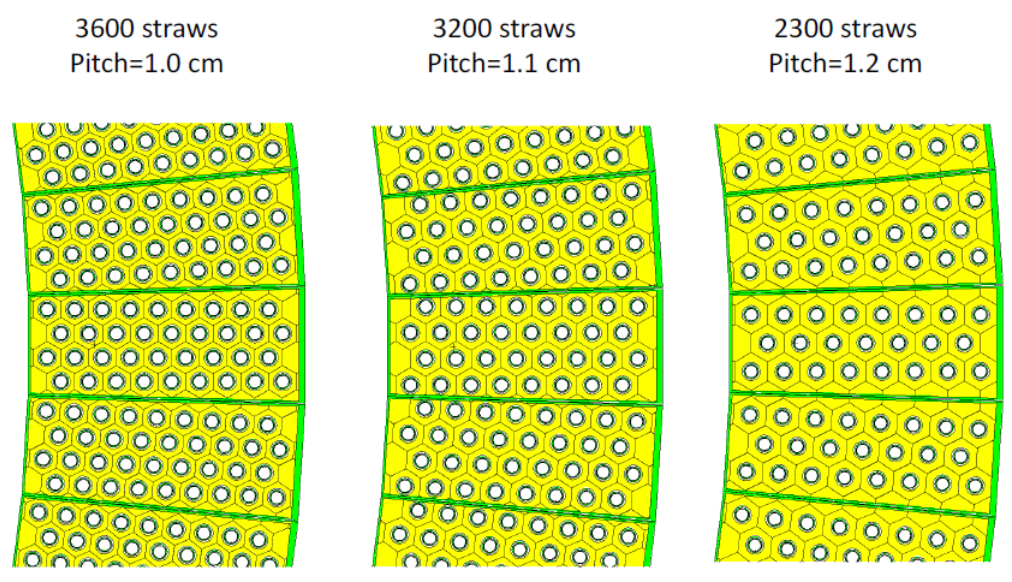

Figure 6. Various wedge and straw geometries. Simulation by Proportional Technologies Inc. 


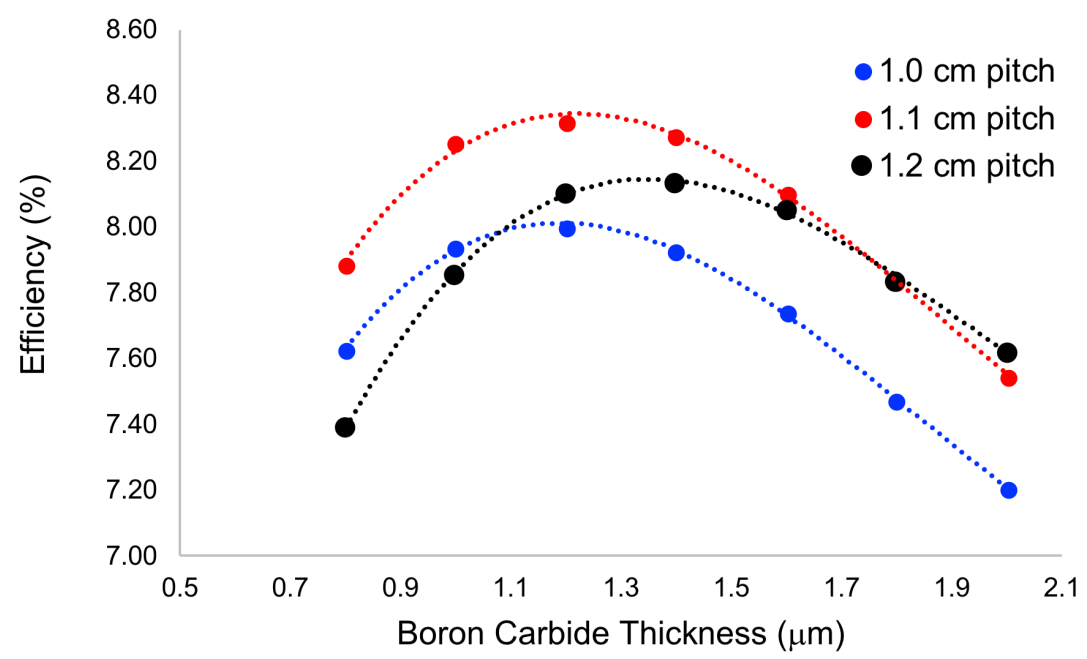

Figure 7. Efficiency as a function of boron carbide coating thickness for the three different straw geometries Simulation by Proportional Technologies Inc.

\subsection{DETECTOR GAMMA-RAY SENSITIVITY}

The gamma radiation field in proximity to spent fuel is a significant challenge for the design of a tomographic neutron imaging system. An unshielded PWR fuel assembly with an exposure of $40 \mathrm{GWd} / \mathrm{MTU}$ and cooling time of one year produces a gamma dose rate of over 1,000 R/h at a distance of $50 \mathrm{~cm}$. As a result, minimizing the gamma-ray sensitivity of the detector is essential to the viability of the imager design.

In any detector, gamma-rays can transfer energy to electrons via Compton scattering. In the boron straw detectors, this can take place in the detector walls or the fill gas, yielding high energy electrons that produce ionization as they traverse the fill gas of the detector. The vast majority of gamma-ray interactions produce small pulses that correspond to a few tens of kiloelectronvolts of ionization at most. If the gamma-ray rate is sufficient, then many such small pulses can pile up to produce a detector response comparable in magnitude to neutron pulses. Note that counts originating from pulse pileup can be minimized by reducing the detector volume associated with each channel of readout and by using readout circuitry that is as fast as possible to minimize the time window over which pileup can occur. A separate source of gamma sensitivity comes from large pulse height interactions of single gamma rays that deposit a substantial fraction of their energy in the detector. These low probability, large pulses represent a fundamental limitation on detector sensitivity to gamma rays.

To experimentally determine the sensitivity of the boron straw detectors to high dose rates, measurements were performed at the ORNL Radiological Assessment Systems for Consequence Analysis cesium irradiator facility with a prototype neutron detector that consisted of 23 straws. A photograph of the detector mounted on the irradiator table is shown in Figure 8. To emulate exposure to comparable dose rates to those originating from spent fuel, the detector was exposed to the highest possible dose rates available from the ${ }^{137} \mathrm{Cs}$ irradiator. This was achieved by positioning the irradiator table a distance $78 \mathrm{~cm}$ from the irradiator opening. At this distance, the source illuminated approximately one-third of the detector with a dose rate of $432 \mathrm{R} \mathrm{h}^{-1}$, a configuration equivalent to exposing eight straws in their entirety to $432 \mathrm{R} / \mathrm{h}$. Measurements at additional dose rates of $105.1 \mathrm{R} / \mathrm{h}$ and $22 \mathrm{R} / \mathrm{h}$ were achieved by configuring the irradiator with the $\times 4$ and $\times 20$ attenuators, respectively. 


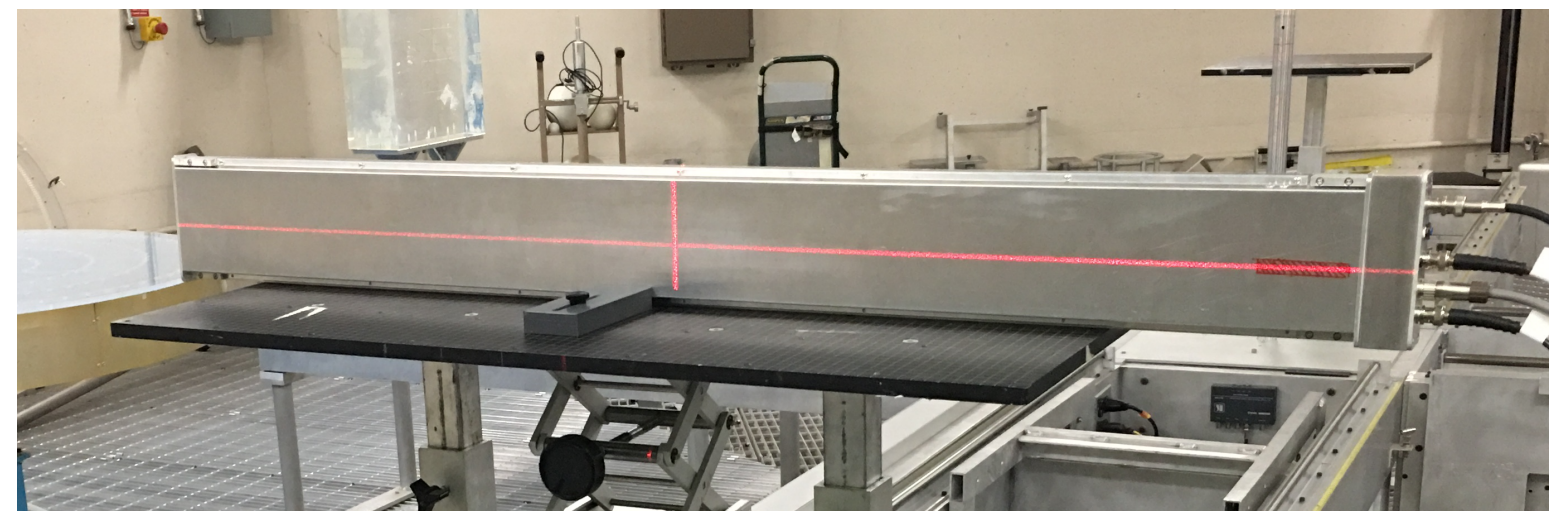

Figure 8. The prototype detector during irradiation experiments. The gamma irradiation was centered on the crosshairs.

To minimize the effects of pulse pileup, a fast, bipolar shaping amplifier circuit was developed. The bipolar output pulse of the circuit has zero net area, ensuring that at high rates, the detector baseline does not drift from ground. The speed of the pulse, with a leading-lobe full width of $50 \mathrm{~ns}$, minimizes the probability of pulse pileup. An example neutron output pulse from the circuit is shown in Figure 9.

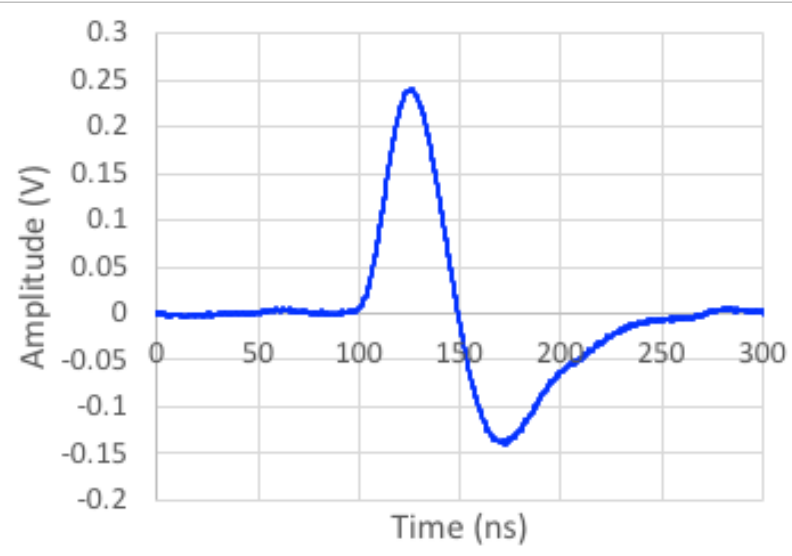

Figure 9. A typical neutron pulse.

The response of the detector baseline to gamma irradiation by applied fields of $0,22,105$, and $430 \mathrm{R} / \mathrm{h}$ is shown for sequential $15 \mu$ s oscilloscope traces in Figure 10. 


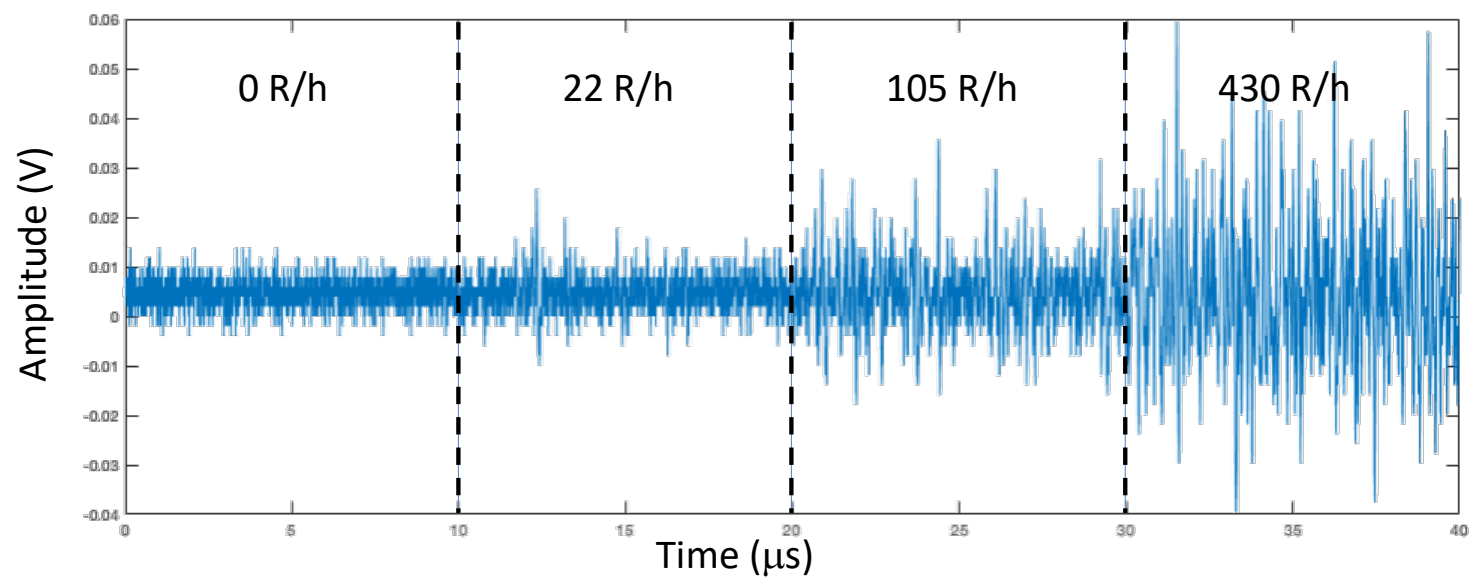

Figure 10. Oscilloscope traces corresponding to the four gamma dose rates to which the detector was exposed.

At the highest radiation fields, the detector output shows significant excursions from baseline. To determine whether these excursions consist primarily of large single pulses or pileup of many small gamma-ray pulses, a high-statistics simulation of detected pulse heights due to irradiation of the detector by $662 \mathrm{keV}$ gamma rays was performed. Gamma and electron transport were performed using MCNP 5, and the energy deposition in the detector gas was recorded using the F8 tally. In total, $7.13 \times 10^{10}$ histories were simulated; simulation of 1 second of irradiation in the configuration with a dose rate of $432 \mathrm{R} / \mathrm{h}$ is equivalent to $9.55 \times 10^{10} 662-\mathrm{keV}$ gamma rays. The resulting calculated pulse height spectrum is shown in (a) of Figure 11. For comparison, the calculated pulse height spectrum corresponding to neutrons is shown in (b).
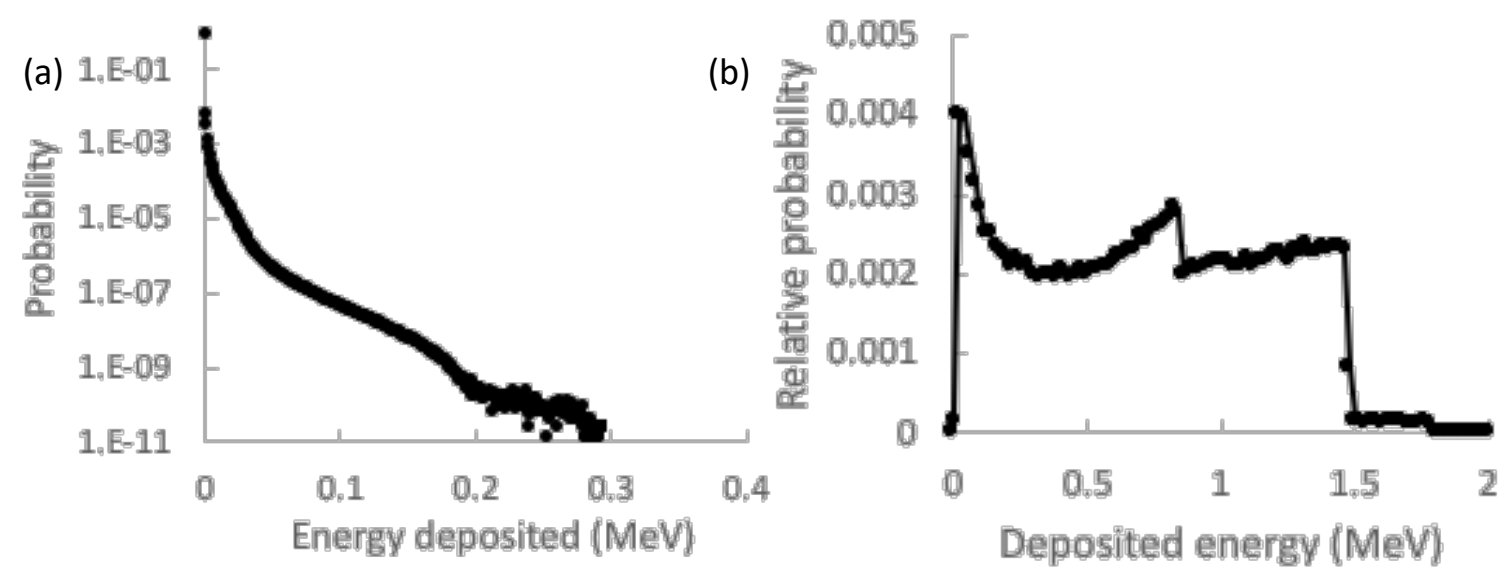

Figure 11. Pulse height spectrum vs. energy (MeV) from an MCNP simulation of (a) a beam of $662-\mathrm{keV}$ photons incident on a single detector and (b) the corresponding pulse height spectrum for neutrons.

Although the neutron spectrum stretches to significantly higher energy, the essential parameter is the relative efficiency of a threshold that excludes almost all pulses induced by gamma rays. Consequently, the pulse height spectrum (together with the simulated neutron pulse height spectrum) was used to calculate the expected count rate as a function of threshold for both gamma rays and neutrons. Using an empirical conversion of $300 \mathrm{mV} / \mathrm{MeV}$ in the straw detector, the simulated curves were plotted along with measured data for dose rates of $0 \mathrm{R} / \mathrm{h}, 22 \mathrm{R} / \mathrm{h}$, and $430 \mathrm{R} / \mathrm{h}$ and are shown in Figure 12. Here, the calculated count rates reproduce the measured count rates at $430 \mathrm{R} / \mathrm{h}$ but overpredict the count rates for lower discriminator thresholds at the $22 \mathrm{R} / \mathrm{h}$ dose rate. This discrepancy is most likely due to differences 
in the experimental and modeled spectra from the irradiator, where the former includes down-scattered energies from the collimator and attenuators. However, the simulations suggest that the detectors are either at or close to their fundamental limitation set by low-probability, large amplitude interactions of single gamma rays. As a result, only configurations with a maximum dose rate less than $500 \mathrm{R} / \mathrm{h}$ will be considered for this application. Additionally, to ensure that the amount of detector pileup in imaging measurements is no more than that encountered in the high dose rate measurements, the detectors will be instrumented with a preamplifier per row of eight straws. Unfortunately, this number of preamplifiers corresponds to a factor of 3 increase compared to the originally envisioned single preamplifier per set of 23 straws.

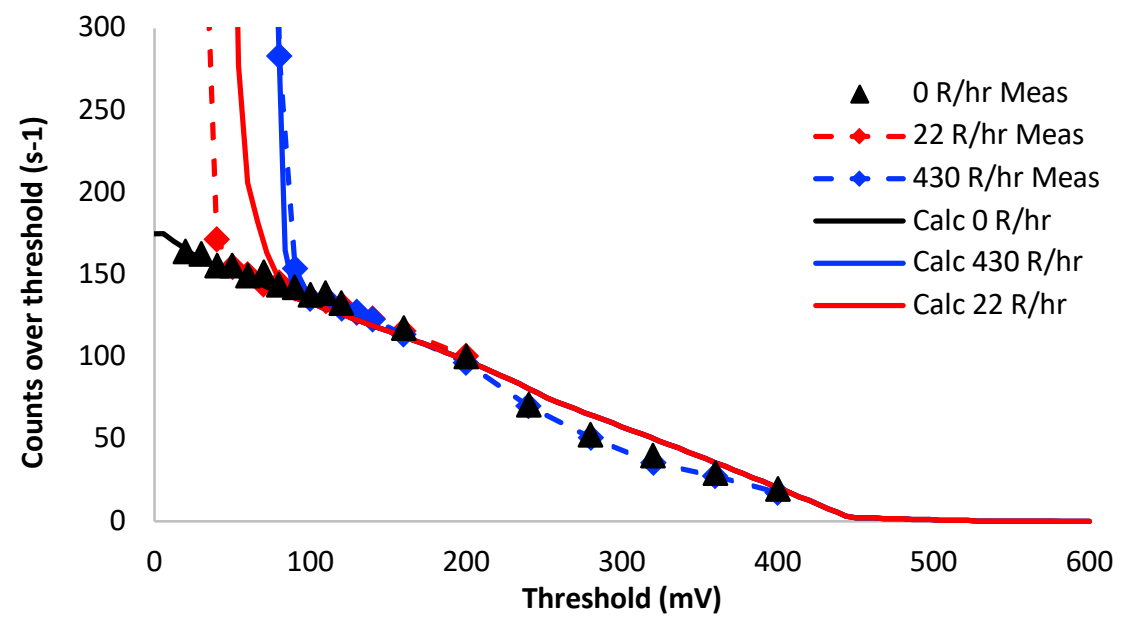

Figure 12. Measured (solid) and simulated (dashed) counts per second in a single detector module as a function of detector threshold for different dose rate exposures.

\section{SIMULATION DESIGN STUDY}

This section documents the results of radiation-transport simulations intended to optimize the performance of the prototype imager. In this regard, "optimal" means minimizing the time to resolve individual fuel pins. In practice, the optimal imager is the one that has the highest SNR for measurements of fuel assemblies while maintaining gamma dose rates that are tolerable to the detectors. In addition, the imager will have to achieve this performance with a reasonable overall size. For the optimization process, gamma dose rates and neutron responses were calculated for a survey of hundreds of collimator configurations whose parameters (such as collimator thickness and slit width) approximately spanned the range of reasonable values. In particular, 16 different combinations of stainless steel and borated polyethylene thicknesses were simulated for each of 32 different slit widths for a total of 512 collimator configurations. These configurations included nine parallel slits spanning $1-5 \mathrm{~mm}$ in $0.5 \mathrm{~mm}$ intervals and 23 tapered slit combinations with inner dimensions of $1 \mathrm{~mm}, 2 \mathrm{~mm}, 2.5 \mathrm{~mm}$, and $3 \mathrm{~mm}$ and outer dimensions that ranged from $0.5 \mathrm{~mm}$ more than the inner dimension to $5 \mathrm{~mm}$ in $0.5 \mathrm{~mm}$ intervals.

For evaluation of the gamma-ray response, dose rates were calculated for each detector of each configuration. Then, the aggregate of these results was used to understand the scaling of dose rate with collimator parameters. After comparison of calculated dose rates with results from prototype detector testing, configurations with dose rates that can be tolerated by the detectors were identified.

For evaluation of the neutron response, components of the response of individual detectors and their associated collimator slits to neuron sources were identified and simulations to quantify each component were performed. These components included 
- a "direct" component consisting of direct illumination of the detector by the source, unimpeded by the collimator slit;

- an "edge" component where the slit wall either partially or fully blocks the direct path to the detector but average paths from the source to the detector through the collimator are substantially smaller than the full collimator thickness;

- "inter-detector scattering," where neutrons traverse one slit, then scatter into different detectors where they are subsequently detected; and

- "collimator penetration," where neutrons traverse the collimator similarly to a solid (but lowerdensity) collimator.

Separation of the response into constituent components was useful for two reasons. First, studying the components individually made it possible to understand which components provided the primary limitations on performance. Second, separating the response into constituent components aided development of a simplified, rapid calculation to estimate the neutron response of each detector to a source element placed at each point in the field of view (FOV). For evaluation of the collimator configurations, the estimated response was used to calculate an SNR ratio for each configuration (in the future, the estimated response will also be used as the forward projection of an iterative image reconstruction code). This SNR was defined in such a way that it accounts for both efficiency (counting statistics) and resolution so that its value is representative of the ability of an imager configuration to image a single missing fuel pin. Then, SNR was used to rank imager configurations, and imager configuration having a favorable combination of SNR and dose rate were identified.

To enable deployment and handling at fuel-cycle facilities, the imager is envisioned to be enclosed within a container. The size of the imager and its container are constrained by practical considerations, but appropriate fuel-cycle facilities are prepared to handle nuclear fuel transport casks or cask-like containers. As a result, two commercial casks were used as exemplar containers to constrain the imager dimensions: (1) the GNS Castor V/52 transport cask (without the fuel basket), which has an inner diameter of $148 \mathrm{~cm}$ and an outer diameter of $244 \mathrm{~cm}$, and (2) the Fuel Solutions TS125 transport cask, which has an inner diameter of $170.2 \mathrm{~cm}$ and an outer diameter of $197.4 \mathrm{~cm}$ [12], [13]. Each imager configuration considered in the survey of designs was constrained to fit within one of the two casks. In the radiation-transport simulations, the appropriate cask was included in the model to account for reflection from an outer vessel and the distance between the outer diameter of the imager and the inner diameter of the cask was filled with borated polyethylene shielding to lessen reflection of neutrons from the cask back into the detectors. The 16 different combinations of thicknesses of stainless steel and borated polyethylene used in the simulations are shown in 
Table 1, along with the associated transport cask and outer shielding thicknesses.

For the baseline imager design, a three-dimensional rendering (with a cutaway for clarity) of the imager housed within the GNS transport cask is shown in Figure 13. 
Table 1. Different combinations of stainless steel and borated polyethylene thicknesses being considered for the simulations going forward based on size constraints of two commercially available transport casks.

\begin{tabular}{|c|c|c|c|c|}
\hline $\begin{array}{c}\text { Associated } \\
\text { transport cask }\end{array}$ & $\begin{array}{c}\text { Collimator } \\
\text { stainless steel } \\
\text { thickness } \\
\text { (cm) }\end{array}$ & $\begin{array}{c}\text { Collimator borated } \\
\text { polyethylene thickness } \\
\text { (cm) }\end{array}$ & $\begin{array}{c}\text { Total collimator } \\
\text { thickness } \\
\text { (cm) }\end{array}$ & $\begin{array}{c}\text { Outer borated } \\
\text { polyethylene } \\
\text { shield } \\
\text { (cm) }\end{array}$ \\
\hline GNS & 5 & 35 & 40 & 5.8 \\
\hline GNS & 10 & 30 & 40 & 5.8 \\
\hline GNS & 6 & 36 & 42 & 3.8 \\
\hline GNS & 5 & 38 & 43 & 2.8 \\
\hline GNS & 6 & 37 & 43 & 2.8 \\
\hline GNS & 7 & 36 & 43 & 2.8 \\
\hline GNS & 5 & 40 & 45 & 0.8 \\
\hline GNS & 6 & 39 & 45 & 0.8 \\
\hline GNS & 7 & 38 & 45 & 0.8 \\
\hline GNS & 8 & 37 & 45 & 0.8 \\
\hline GNS & 10 & 35 & 50 & 0.8 \\
\hline Fuel Solutions & 5 & 45 & 50 & 6.9 \\
\hline Fuel Solutions & 10 & 40 & 53 & 6.9 \\
\hline Fuel Solutions & 5 & 48 & 55 & 3.9 \\
\hline Fuel Solutions & 5 & 50 & 55 & 1.9 \\
\hline Fuel Solutions & 10 & 45 & & 1.9 \\
\hline
\end{tabular}

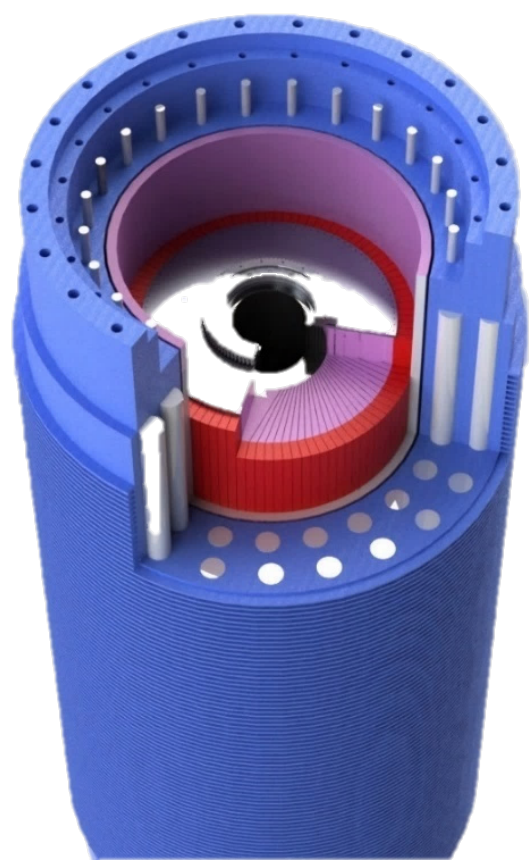

Figure 13. Conceptual three-dimensional rendering of the baseline imager mounted in a GNS transport cask. 


\subsection{GAMMA DOSE RATE SIMULATIONS}

The purpose of the dose rate simulations was to calculate expected gamma dose rates to the neutron detectors for a survey of collimator configurations that span the expected parameter space. Then, configurations that limit the gamma dose rate to a manageable level were identified. Contrary to initial estimates by PTI, manageable dose rates were identified in Section 4.2 to be less than or equal to about $500 \mathrm{R} \mathrm{h}^{-1}$. At this dose rate, the detectors can retain most of their efficiency while operating in a domain where the fraction of detected counts originating from gamma-induced signals is small compared to true neutrons.

For the simulations, the gamma source was taken to be a "worst case" fuel having a high burnup (40 GWd/MTU) and a short cooling time (1 year). The gamma source term for this fuel was generated by the ORIGEN 2.2 isotope generation and depletion code, and the resultant emission rate of $4.03 \times 10^{16}$ photons s $^{-1}$ for a fuel assembly was appropriately apportioned between 24 unequally sized energy bins spanning 0-20 MeV. For the simulations, this gamma source was taken to be distributed evenly over the fuel elements of a $17 \times 17$ fuel assembly. Then, the fuel assembly was combined with each of the 512 collimator configurations comprising the parameter survey and gamma transport performed using the MCNP simulation code. To calculate gamma dose, interactions within the P-10 fill gas of the detectors were tallied using the F6 tally $\left(\mathrm{MeV} \mathrm{g}^{-1}\right.$ photon $\left.^{-1}\right)$, and then converted to rem per hour using the conversion:

$$
\frac{R}{\text { hour }}=\frac{F 6\left[\frac{\mathrm{MeV}}{\mathrm{g} * \text { photon }}\right] * 4.02507 \times 10^{16}\left[\frac{\text { photons }}{\text { second }}\right] * 3600\left[\frac{\text { seconds }}{\text { hour }}\right]}{6.24 \times 10^{7}\left[\frac{\mathrm{MeV}}{\mathrm{Rad} * \text { gram }}\right] * 0.876\left[\frac{\mathrm{Rad}}{\mathrm{R}}\right]}
$$

Here, the photon intensity $4.02507 \times 10^{16}\left[\frac{\text { photons }}{\text { second }}\right]$ for a fuel assembly was scaled from

$$
8.9946 \times 10^{15} \frac{\text { photons }}{\text { second } * 0.1 M T U} * 0.45 \frac{M T U}{\text { fuel assembly }}=4.02507 \times 10^{16}\left[\frac{\text { photons }}{\text { second }}\right] .
$$

In addition to identifying configurations that have manageable dose rates for the worst-case fuel, examination of the simulated dose rates was desirable to understand the straw-to-straw variations of dose rate in a given geometry. Measured data will include some portion of gamma-induced counts, so systematic variations in dose rate could produce associated variations in gamma-induced counts from detector to detector. Therefore, it is advantageous to be cognizant of the magnitude of variations in dose rate between detectors.

In this context, Figure 14 shows a heat map of the gamma dose rates seen at each straw in the baseline imager. Recall that the collimator of the baseline design consists of $10 \mathrm{~cm}$ stainless steel and $30 \mathrm{~cm}$ borated polyethylene with $3 \mathrm{~mm}$ wide parallel slits. For the baseline imager design, detector-averaged gamma dose rates vary by about a factor of 3 from detectors that view the portion of the collimator with the smallest slit density and correspondingly highest areal density paths through the collimator to detectors that view the portion of the collimator with the largest slit density and correspondingly lowest areal density paths through the collimator. Within detectors, straws closest to the slit openings observe the highest dose rates, and the dose rate drops by about a factor of 2 from the side of the detector nearest the slits to the side of the detector farthest from the slits. Importantly, the highest dose rates calculated for the baseline imager (averaged over a detector) do not exceed $500 \mathrm{R} / \mathrm{h}$, so the baseline imager potentially constitutes a viable design. 


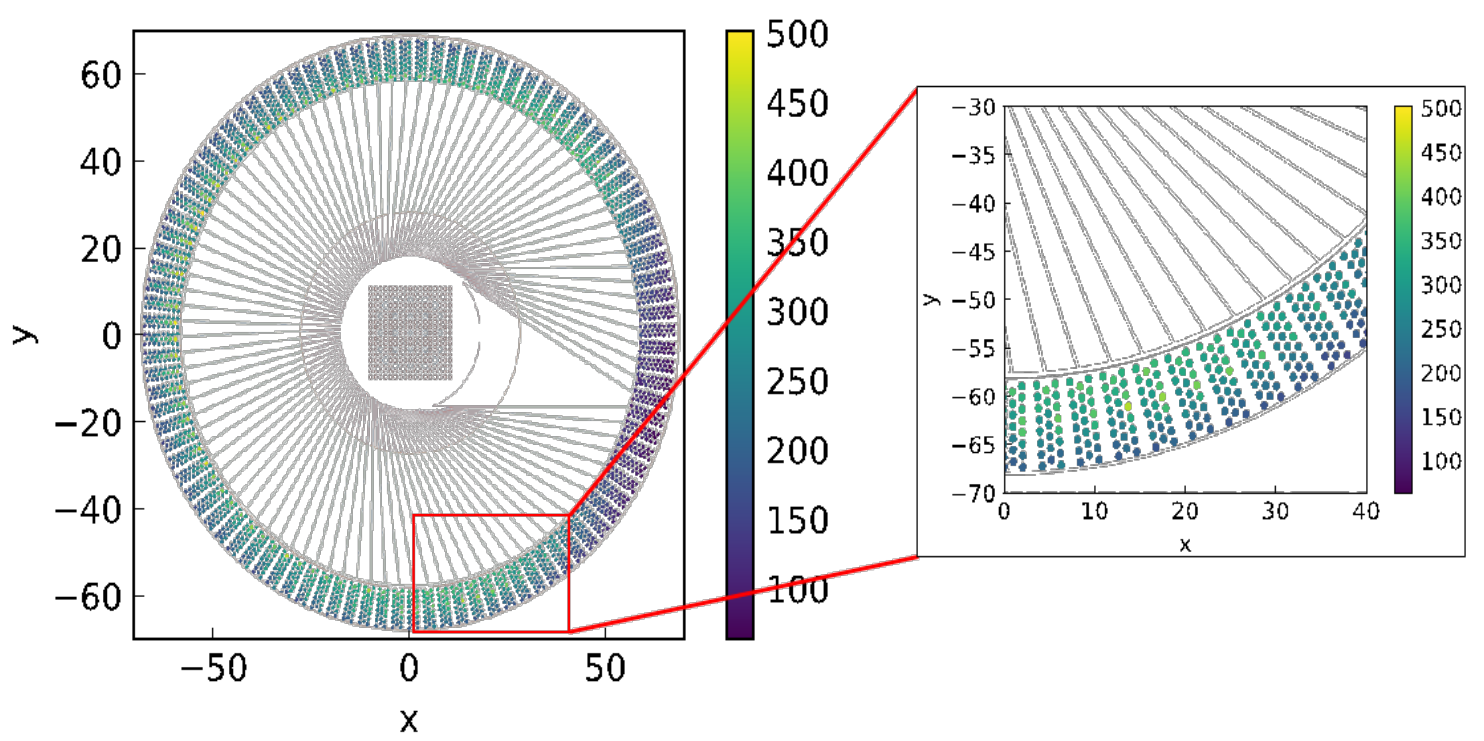

Figure 14. Map of gamma dose rates seen at all the detector straws for a collimator with $10 \mathrm{~cm}$ stainless steel, $30 \mathrm{~cm}$ borated polyethylene, and $3 \mathrm{~mm}$ parallel slits with a $40 \mathrm{GWd} / \mathrm{MTU}$ burnup fuel with 1 year cooling time as the source term.

In general, collimator configurations with small slit widths see minimal differences in dose rates between detectors, but as slit widths grow, so do the differences in dose rate. In Figure 15, the minimum and maximum detector average dose rates are shown as a function of the configuration average dose rate for all simulated configurations.

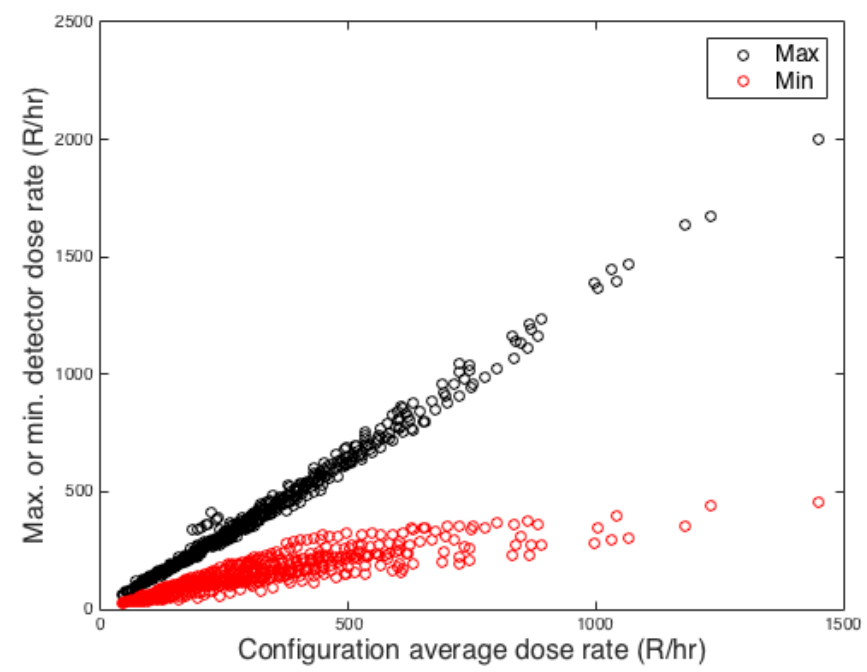

Figure 15. Dose rates of the detectors with the (black) maximum and (red) minimum dose rate for each collimator configuration compared to the configuration average dose rate.

Note that the maximum detector average dose rate is approximately 1.33 times the configuration average dose rate for all collimator configurations, independent of the areal density of the collimator. The minimum detector dose rate changes less from configuration to configuration. This is because the minimum dose rate is associated with detectors that view the nearly solid portion of the collimator, and as slit widths change, the collimator areal density viewed by these detectors changes less. 
In addition to evaluating detector-to-detector variation in dose rates, examination of the results of dose rate simulations was desirable to engender design intuition and identify "rules of thumb" that capture scaling of dose rates with simple physical quantities, such as the average areal density of the collimator. Identification of rules of thumb then enables ready comparison between collimator configurations and supports evaluation of configurations that may be beyond the bounds of the calculated configurations.

With this purpose in mind, the maximum detector average dose rate was examined. While the collimator rotates, each detector in turn is exposed to the maximum dose rate, so this dose rate dictates the required gamma insensitivity of the detectors. The maximum calculated dose rates spanned the range from $60 \mathrm{R} / \mathrm{h}$ to $2,000 \mathrm{R} / \mathrm{h}$, with lower values corresponding to configurations with thicker collimators and narrower slits. The scaling of dose rates with collimator parameters can be understood by plotting the dose rate per centimeter of steel as a function of the average collimator areal density (in $\mathrm{g} / \mathrm{cm}^{2}$ ). This plot is shown for the totality of simulations in Figure 16 and indicates that the dose rate drops approximately exponentially with larger areal densities (that result from thicker collimators or narrower slits) with a $1 / e$ length of $16 \mathrm{~g} / \mathrm{cm}^{2}$. The dose rates are plotted per centimeter of stainless steel because for two collimators having different thicknesses of steel but the same areal density, the average density of the steel portion is reduced, and gamma streaming is increased, for the configuration with the thicker steel section by the relative thickness of steel.

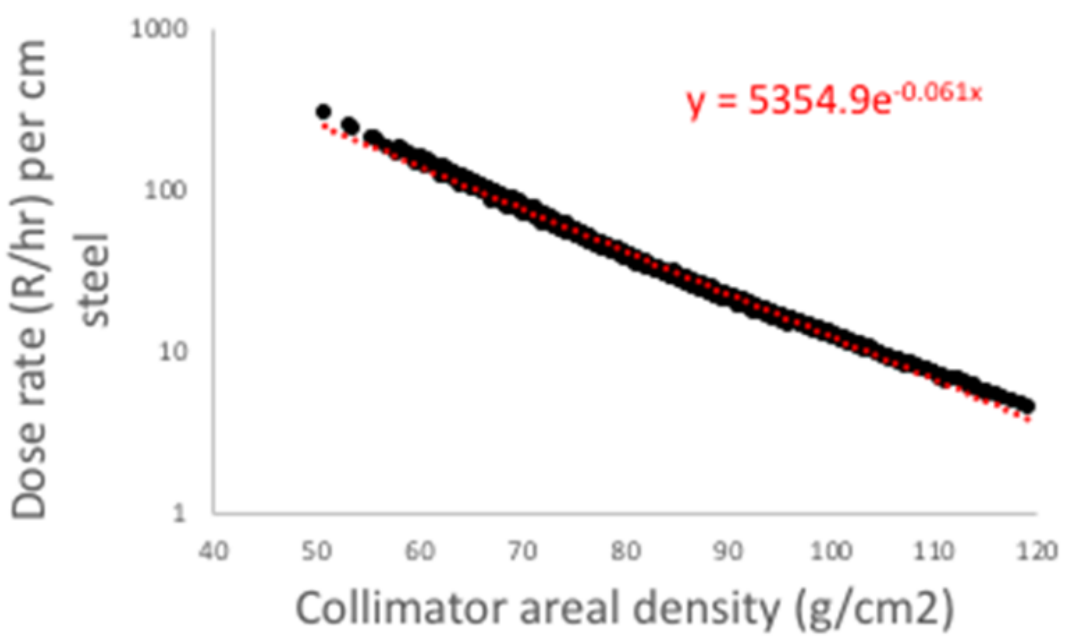

Figure 16. Maximum detector gamma dose rates per centimeter stainless steel for all 512 cases as a function of the collimator areal density.

\subsection{NEUTRON RESPONSE SIMULATIONS}

The purpose of the neutron response simulations was to calculate the expected performance of the imager for detecting the absence of a single fuel pin for each configuration of a survey of collimator configurations that span the expected parameter space. Ideally, this problem would be addressed by simulating tomographic fast-neutron imaging measurements for each collimator configuration, performing image reconstruction on the simulated data, and evaluating the SNR in the reconstructed images. However, this approach would involve a prohibitive level of effort for each collimator configuration. Instead, an approach was adopted where collimator configurations could be evaluated independently of reconstruction algorithms by defining an SNR that captures the quality of data that feeds the image reconstruction. Then, configurations were evaluated by calculating the value of the SNR and more favorable ones were identified. 
To calculate the SNR, it was desirable to know the imager response over the entire FOV on a millimeterlength scale for each projection angle. To reduce the computational burden associated with calculating the SNR for each configuration to a manageable level, an approximate imager response was developed. This approximate response was built from a number of constituent response components that could be identified in analysis of simulations (e.g., direct response, edge response, collimator penetration, and inter-detector scattering). This approximate response can be computed sufficiently fast that it can be calculated on the millimeter-length scale over the entire imager FOV for each configuration and each projection angle. In addition to forming the basis of the SNR calculation, the approximate imager response will eventually be used as the forward projection of an iterative reconstruction code.

For the present work, neutron simulations were performed using MCNP 6, and the F4 tally function was used to generate a reaction rate for neutrons captured by ${ }^{10} \mathrm{~B}$ contained in the boron carbide $\left(\mathrm{B}_{4} \mathrm{C}\right)$ in each of the 100 wedge-shaped detectors. The F4 neutron tally by itself is a track-length estimate of the average cell flux. If a particle of weight $W$ makes a track of length $T(\mathrm{~cm})$ within a specified cell of volume $V$ $\left(\mathrm{cm}^{3}\right)$, the contribution to the flux in the cell is given by Equation (1) below.

$$
W \frac{T}{V}
$$

The sum of these contributions is reported as the F4 tally in the MCNP output. The units of the F4 tally outputs are in particles per centimeter squared. Since the quantity of interest is the reaction rate of neutrons captured on boron carbide, a tally multiplier was used to convert the F4 tally to a reaction rate. The tally multiplier card, specified by the mnemonic FM, multiplies the tallied quantity by the cross section of interest to give the reaction rate per number of source particles, as shown in Equation (2):

$$
\text { neutron capture reactions per nps }=F 4 N \sigma_{a b s} V \text {, }
$$

where $N$ is the number density of boron carbide $\left[\frac{\text { atoms }}{\text { barn cm }}\right]$ ], $\sigma_{a b s}$ is the absorption cross section of the boron carbide in barns, and $V$ is the volume of boron carbide a single detector straw in cubic centimeters.

This section presents the results of simulations used to understand the imager response, identify the magnitude and scaling of different effects, and develop an approximate imager response. This section also details the definition and evaluation of SNR and presents results for the survey of collimator designs.

\subsubsection{The "direct" and "edge" components of the response}

Some aspects of understanding the response of the collimator were aided by simplifying the collimator configuration. In particular, the behavior of a collimator with a single slit was studied to eliminate complications associated with neutrons traversing neighboring slits and then scattering into the detector of interest. Supposing the behavior of a single slit was understood, the response of multiple slits can be understood as the sum of the responses to individual slits. The simplified collimator configuration is shown schematically in Figure 17. 


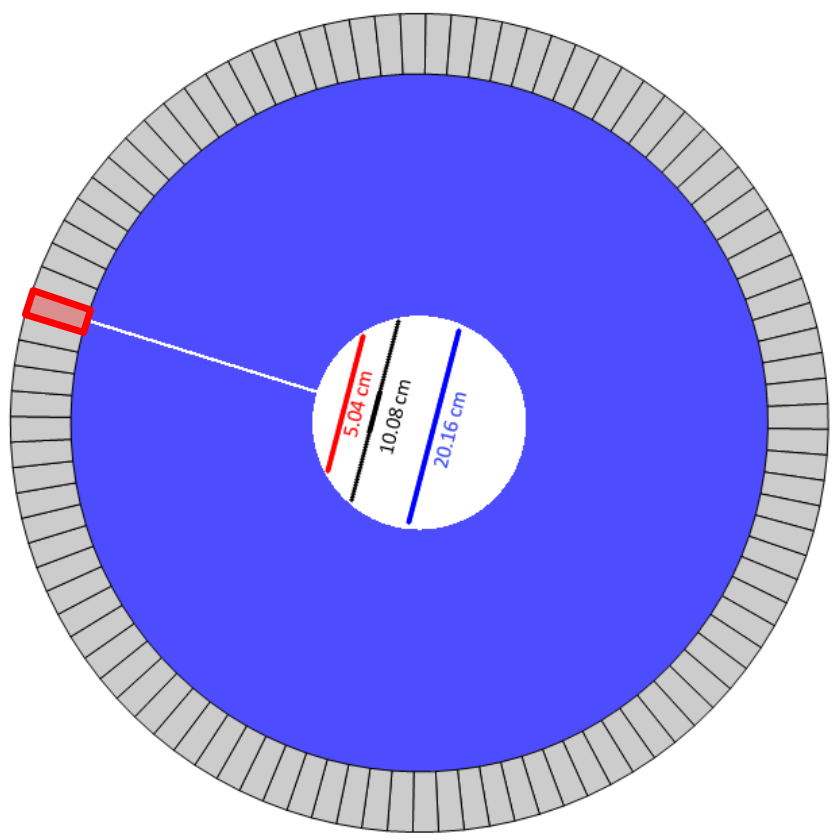

Figure 17. The simplified collimator configuration with a single slit. The detector at the terminus of the slit is highlighted red.

The response to the single slit was investigated by simulating a ${ }^{244} \mathrm{Cm}$ line source at a number of positions in the FOV and recording the response of the detector at the terminus of the slit. To investigate the direct and edge components of the response, the line source was positioned at a number of locations along a line perpendicular to the centerline of the single slit at three different distances from the slit opening: 5.04, 10.08 , and $20.16 \mathrm{~cm}$. For the simulations associated with the $10.08 \mathrm{~cm}$ distance from the slit opening, 118 different source positions were simulated spanning the distance from the slit centerline of $-35 \mathrm{~mm}$ to $+35 \mathrm{~mm}$ in $1 \mathrm{~mm}$ steps and further extending to $-150 \mathrm{~mm}$ and $+155 \mathrm{~mm}$ from the slit centerline in $5 \mathrm{~mm}$ steps. These positions are shown in Figure 17 using black markers (although the markers overlap to appear as a line). When taken together, the 118 source positions will be referred to as a "source transit" across the slit at a distance of $10.08 \mathrm{~cm}$ because the results are similar to what would be measured if a source were moved along a path transiting the slit opening. Source transits at $10.08 \mathrm{~cm}$ from the slit, each consisting of 118 points, were also simulated for slit widths of $0.0,1.0,1.5,2.0,2.5,3.0,3.5,4.0,4.5$, and $5.0 \mathrm{~cm}$. The collimator configuration for these simulations consisted of $5 \mathrm{~cm}$ stainless steel and $45 \mathrm{~cm}$ borated polyethylene for a total thickness of $50 \mathrm{~cm}$.

For the $5.04 \mathrm{~cm}$ distance from the slit opening, the source transit consisted of simulating 240 different source positions between $-40 \mathrm{~mm}$ and $+40 \mathrm{~mm}$ in $0.5 \mathrm{~mm}$ steps and extending to $-118 \mathrm{~mm}$ and $+120 \mathrm{~mm}$ in $2 \mathrm{~mm}$ steps. These positions are shown in Figure 17 using red markers. Source transits were simulated for slits widths of 0.0 and $5.0 \mathrm{~mm}$. Likewise, for the $20.16 \mathrm{~cm}$ distance from the slit opening, the source transit consisted of simulating 285 different source positions between $-40 \mathrm{~mm}$ and $+40 \mathrm{~mm}$ in $0.5 \mathrm{~mm}$ steps and further extending to $-162 \mathrm{~mm}$ and $+166 \mathrm{~mm}$ in $2 \mathrm{~mm}$ steps. These positions are shown in Figure 17 using blue markers; simulations were performed for slits widths of 0.0 and $5.0 \mathrm{~mm}$.

To illustrate typical data from the simulations, results of a source transit are shown on the left side of Figure 18. In this case, the transit is across a $5 \mathrm{~mm}$ slit at a distance of $20.16 \mathrm{~cm}$ from the slit opening. This plot summarizes 285 simulations, and each point gives the calculated response of the detector at the slit terminus in counts per source neutron for a particular source position identified by its distance from the slit centerline. The distance " 0 " coincides with the slit centerline, and the peak in response in points near 0 correspond to the direct component of the response of the collimator-detector combination. On the 
right of Figure 18, the same plot is shown with the y-axis zoomed to highlight the collimator penetration portion of the response. Note that in this plot, each point corresponds to a separate simulation. The collimator-penetration portion of the response can be approximated by a quadratic, shown by the red line. This fit describes the data sufficiently well that the constant term of the quadratic is taken to be the contribution of neutrons that penetrate the collimator to the detected counts when the source is aligned with the slit.
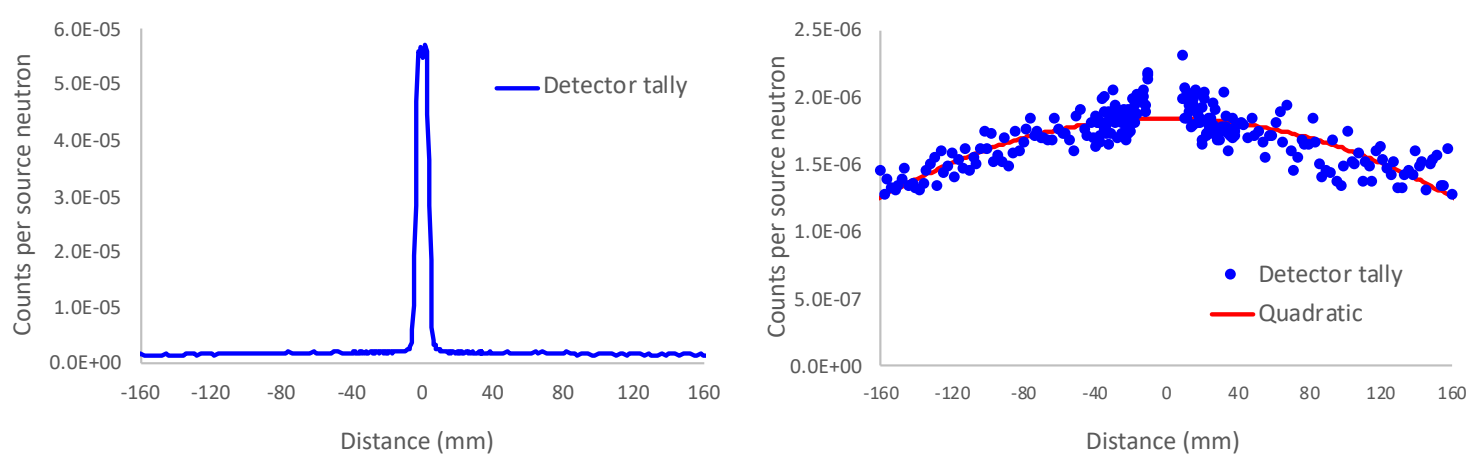

Figure 18. (Left) The response of the detector at the slit terminus to a neutron source at 285 different positions on a line perpendicular to the slit centerline and $20.16 \mathrm{~cm}$ from the slit opening, and (right) the same data displayed to accentuate the portion.

The direct portion of the collimator response can then be deduced as the difference between the value at distance 0 and the constant term of the quadratic. This process was repeated for all simulated source transits. The resulting magnitude of the direct response is plotted as a function of slit width in Figure 19. To compare different source-to-slit-opening distances, each point was multiplied by the ratio of the solid angle of the detector at $10.08 \mathrm{~cm}$ to the solid angle at its given distance. Then, solid angle differences stemming from different source-to-detector distances were eliminated, and the effective solid angle of the detectors should be proportional to the slit width. The linear scaling of the direct component with slit width confirms that the direct component is simply proportional to the solid angle of the slit opening at the detector.

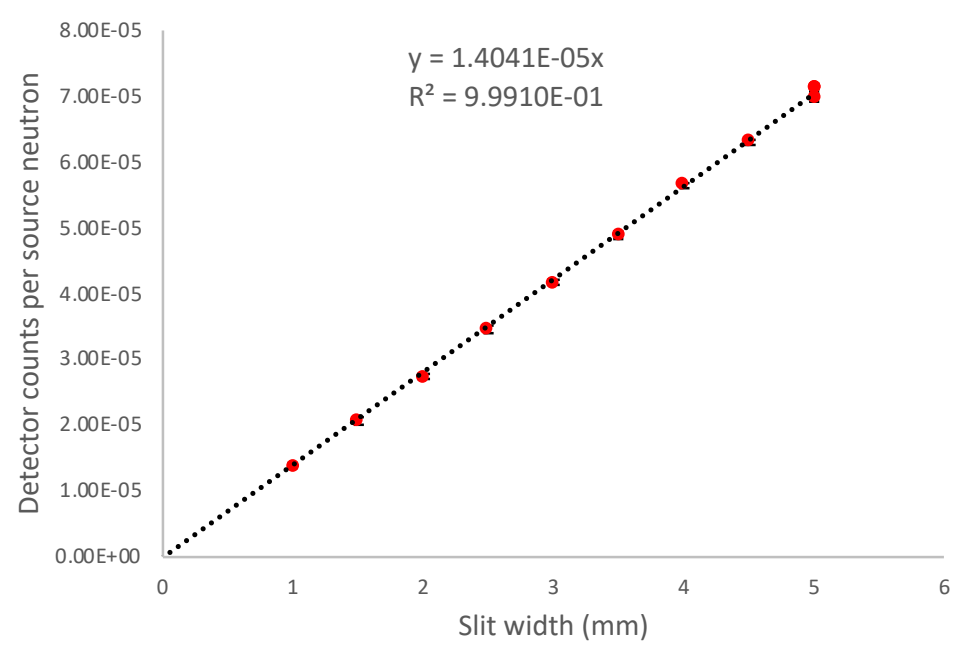

Figure 19. Magnitude of the direct component of detector response as a function of slit width.

The results of source transit simulations were also examined to study the edge component of the detector response. Loosely speaking, the "edge component" of the detector response comprises the portion of the 
response where the value is dropping from the central maximum of the direct response to the value of the collimator penetration where the source is well obscured from the detector at the terminus of the slit.

To discuss the edge component of the detector response, it is helpful to illustrate using a schematic diagram of a single slit. Figure 20 shows a single slit of width $w$ along with the near half of the associated collimator. On this diagram, the $x$ coordinate is taken to be the horizontal direction with zero defined to be the edge of the slit. Then, the vertical line shown in Figure 20 identifies $x=0$. At each height above the slit, there is a projected width of the slit $w_{p}$; one such projection of the slit width is shown by the dotted line. The diagonal line $x=w_{p}$ corresponds to the set of points where the near edge of the slit just obscures the back of the detector. The response of the detector to sources positioned at different points along the line $x=w_{p}$ will be similar because the fraction of the detector shadowed by the slit edge is the same. However, the response will not be identical because the path lengths through the collimator to the detector will be different. The slit-edge region corresponds roughly to points that are between $0 \leq x \leq$ $3 w_{p}$.

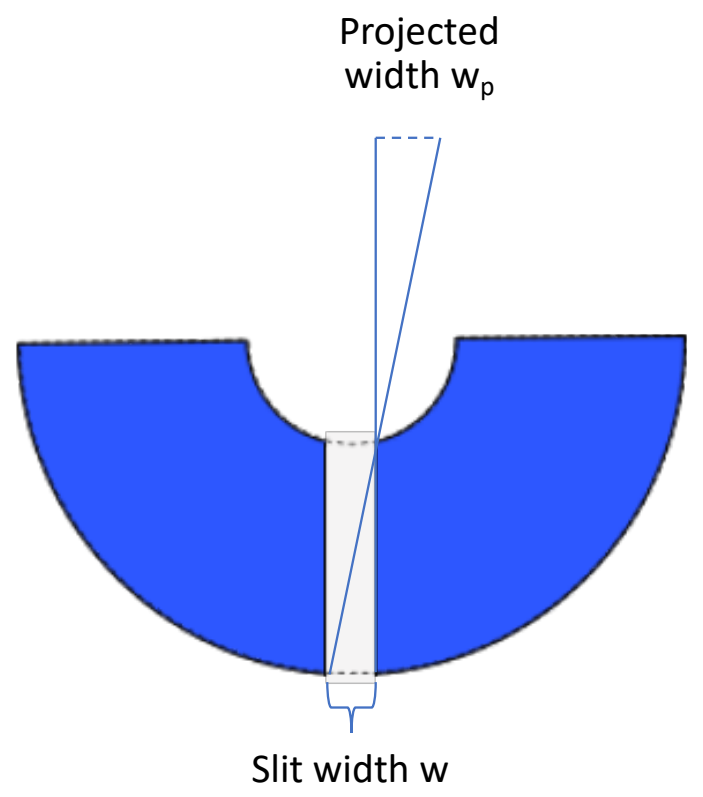

Figure 20. Single slit geometry showing the projection of the slit width in the FOV.

It is desirable to be able to compare the edge response across all slit widths and source-to-slit-opening distances. With this goal in mind, the source transits were manipulated to highlight the edge response. For each source transit, the collimator penetration was subtracted by subtracting the quadratic fit (shown in red in Figure 18). Then, the magnitude of the response was scaled so that the magnitude of the direct component equaled unity. Last of all, the position of the source was given in units of the projected slit width. The data for all source transits are shown together in Figure 21. The source transits at the 5.04, 10.08 , and $20.16 \mathrm{~cm}$ distances are shown by the red, black, and blue markers, respectively. Note that all nine source transits at the $10.08 \mathrm{~cm}$ distance are sufficiently close that the appear to fall on the same trajectory. 


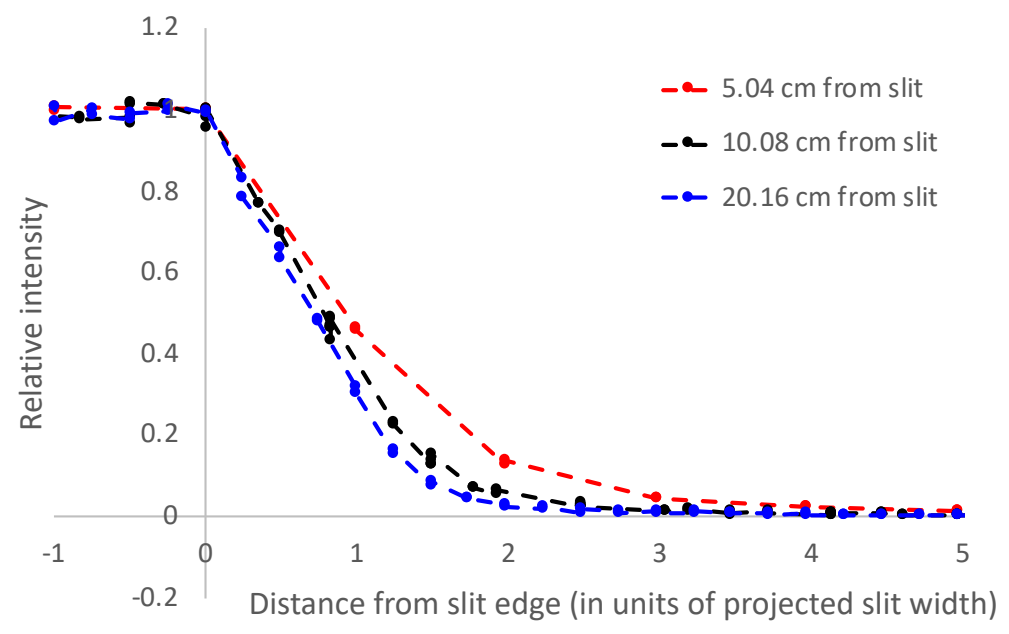

Figure 21. The scaled intensity for the sources at all three distances from the slit opening as a function of the source to slit edge distance in terms of the projected width 'W'.

In contrast, there are more noticeable differences between the responses of different source-to-slit opening distances. This difference originates from the shorter average path length through the wall of the collimator as the source is positioned closer to the slit opening.

\subsubsection{Inter-detector scattering}

One of the biggest factors limiting the resolution of the imager is inter-detector scattering. Inter-detector scattering occurs when neutrons traverse one slit and then scatter from the intended detector to another before being detected. In a true parallel-slit collimator, inter-detector scattering has the same effect as lower spatial resolution because in either case, a fraction of detected neutrons associated with a particular collimated path are misattributed to a nearby path.

To understand the magnitude and extent of inter-detector scattering, simulations were performed in a simplified geometry with 11 detectors present, as shown in Figure 22. For this geometry, a beam of neutrons having the energy spectrum of ${ }^{244} \mathrm{Cm}$ spontaneous fission was directed into the center detector, and the neutron capture reaction rate in each straw was recorded. The results of the simulation are shown overlaid on the schematic diagram of the detector using color scale to indicate the number of neutron detections per straw. Likewise, the relative count rates are shown by detector in the plot in the lower panel of Figure 22. The plot shows that a significant fraction of the neutrons entering the center detector are scattered into the adjacent detectors. The detectors immediately next to the center detector see $\sim 66 \%$ of the counts the center detector sees, and the detectors one away from the center see $\sim 23 \%$. On either side of the central three detectors, the count rate drops off with a mean free path of approximately $3 \mathrm{~cm}$, equivalent to a characteristic length about 0.8 detector widths. 

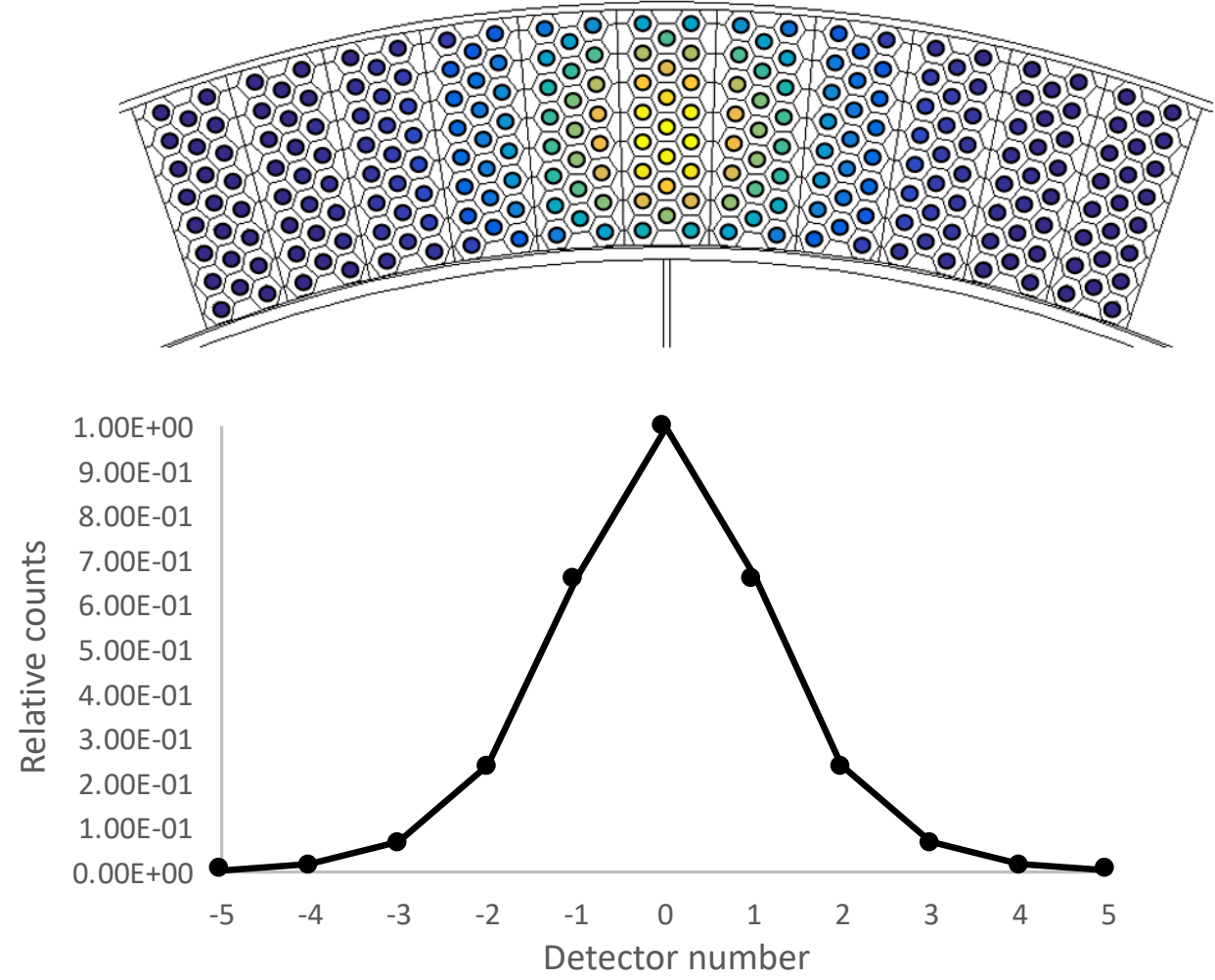

Figure 22. (Above) schematic diagram of 11 detectors with a color scale representation of boron absorption reactions seen in each straw when a beam of ${ }^{244} \mathrm{Cm}$ neutrons is shot to the center detector only. (Below), the relative intensity of boron captures per detector.

The spread in detection locations over several neutron detectors has a rough equivalence to spatial resolution. In a true parallel-slit collimator, the equivalent spatial spread would be at least the full width half maximum in detector pixels multiplied by the slit-to-slit spacing. The non-parallel slits of the ring collimator make the situation more complicated. However, supposing the effect to be similar in magnitude, the equivalent limitation to spatial resolution would be approximately $1 \mathrm{~cm}$. Consequently, inter-detector scattering is a significant limitation on resolution. Given that the resolution is limited by scattering, designs that use slit widths comparable in size to this resolution are likely to be favored.

\subsubsection{Point Spread Functions}

In this section, examples of the simulated response of all 100 detectors in the imager to point neutron sources positioned within the collimator FOV are given. The response of the entire detector to a point neutron source is sometimes referred to as the "point spread function," or PSF. In general, the PSF is used to characterize an imaging system and, more importantly, determines its resolving power [14]. The degree of spreading in the PSF is a measure of the resolution limitation of the imager. In the case of the parallelslit ring collimator, the PSF depends on the position of the source within the FOV.

From a practical point of view, it is too computationally expensive to simulate the PSF for each potential source position within the imager FOV for each imager configuration being considered. Instead, simulations were performed for two representative source positions for each configuration. The two source positions chosen as representative were centered on slit 51 and are shown by the red and blue markers in Figure 23. For the imager designs being investigated, PSFs were generated by simulating a ${ }^{244} \mathrm{Cm}$ line source positioned at these two points for all 512 geometries being considered. 
To compute the PSF, the reaction rate for each neutron detector was tallied, as described at the start of Section 5.2, and plotted against the detector number. The detectors were numbered from 1 to 100 starting at the bottom collimator slit that sits tangential to the fuel assembly cavity and increasing in the clockwise direction as labeled on Figure 23. The resulting PSFs for the red and blue points for the baseline imager are shown in Figure 24. Each PSF has a central peak consisting of a direct contribution and inter-detector scattering. Each PSF also has a generally elevated background consisting of collimator penetration. The blue PSF also shows satellite peaks to either side where other slits can directly view the source position. In the red PSF, the satellite peaks overlap the central peak. As a result, they are not visually distinct, but are nonetheless apparent from the increase in magnitude of the central peak despite the larger distance from the source from the detector. The presence of satellite peaks highlights a complication inherent in the use of non-parallel slits: for many source positions, more than one slit will be able to directly view the source at the same time.

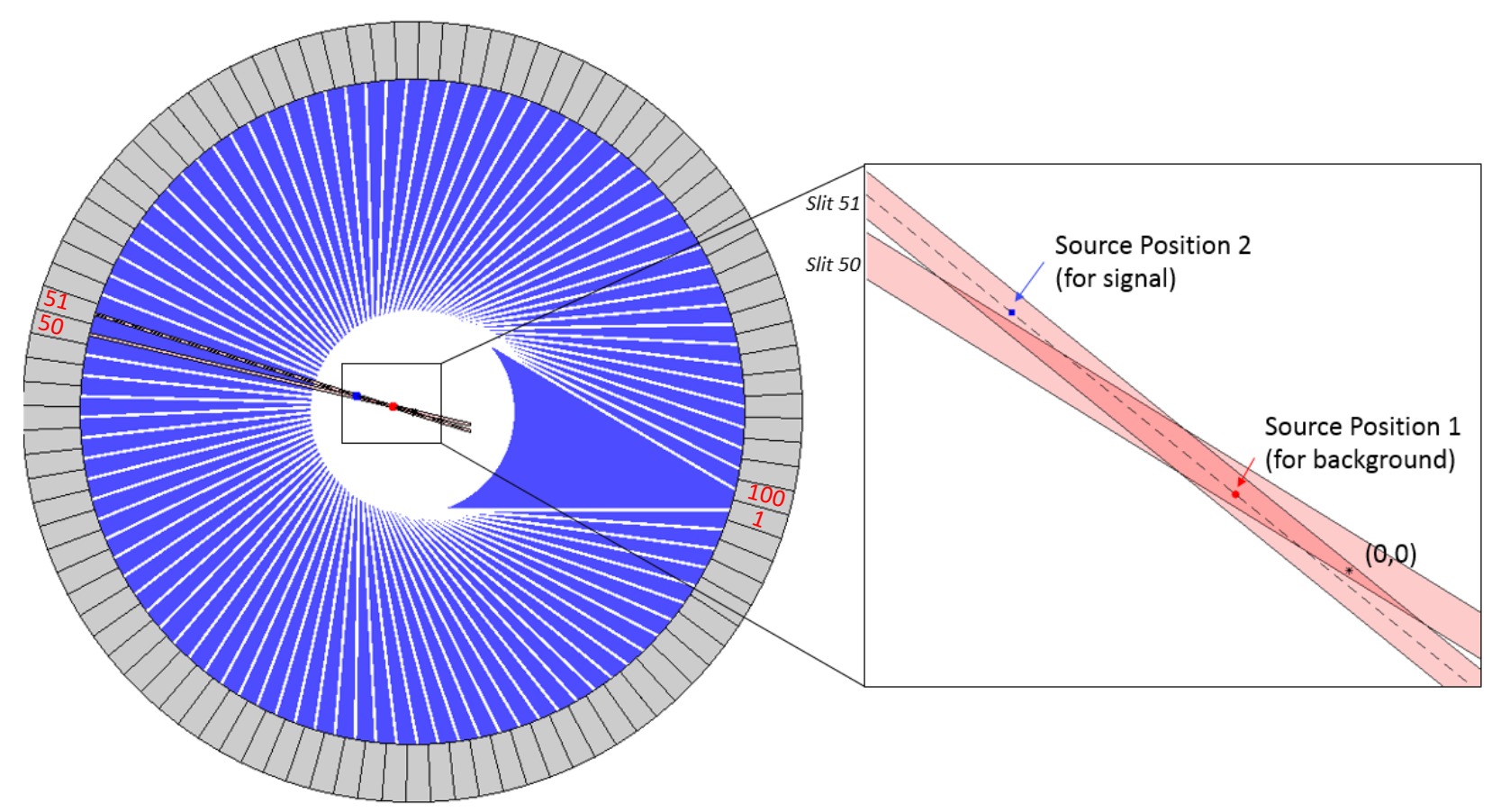

Figure 23. Diagram showing the areas overlapped by the fields of view of slits corresponding to detectors $\mathbf{5 0}$ and 51. The two source positions along slit 51's centerline used to generate the PSFs are also shown here relative to the origin $(0,0)$, which is the center of the fuel cavity. The ${ }^{244} \mathrm{Cm}$ was first placed in the position marked by the red dot to generate PSFs for background quantification and was placed in the position marked by the blue dot outside of the overlap region of the two slits for a second set of simulations to accurately quantify the signal.

The two simulated source positions were chosen to investigate different features of the PSF. For the blue point, the closer proximity of the source to the slit opening was chosen to avoid neutrons having a direct path to the neighboring detector (detector 50), where they can scatter back in to detector 51 and artificially increase the count rate. In the inset of Figure 23, neighboring slit 50 cannot directly view the blue source position. This position was chosen to assess the magnitude of the direct component of the collimator response. (In later analysis, it was discovered that the blue point was not sufficiently far from the neighboring slit for slit widths $>3 \mathrm{~mm}$ ). However, the presence of satellite peaks makes it difficult to use this point to assess collimator penetration. For the red point, its position near the center of the FOV was chosen to assess the magnitude of collimator penetration. This PSF has a clean baseline below detector 40 and above detector 60 without satellite peaks. This baseline portion can be used to assess collimator penetration. 


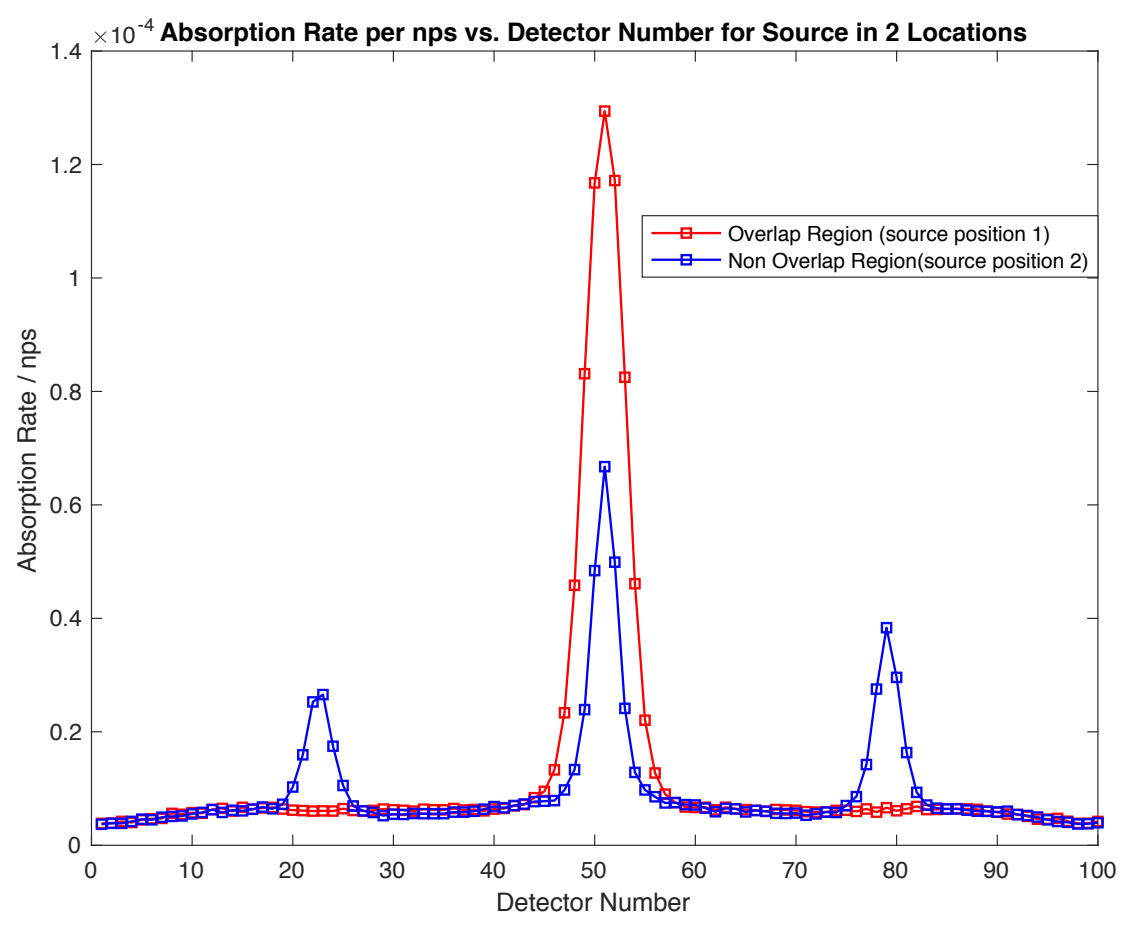

Figure 24. Point spread functions for a ${ }^{244} \mathrm{Cm}$ line source at two different positions within the FOV of the baseline imager (a collimator with $10 \mathrm{~cm}$ stainless steel and $30 \mathrm{~cm}$ borated poly and $3 \mathrm{~mm}$ wide parallel slits).

Later, in the section on SNR, we will use the simulated responses at these positions to interpolate to others. This approach uses the MCNP calculations from two points combined with ray tracing and solidangle scaling to estimate the PSF for each position.

\subsubsection{Collimator penetration}

Although the direct and edge responses of the collimator quantify neutron transmission along preferential paths through the collimator, the collimator penetration portion of the response quantifies the contribution of neutron transmission along non-preferential paths through the collimator. This non-preferential transmission can be thought of as similar to transmission through a solid but lower-density collimator. The collimator penetration contribution to the collimator response is recorded by the non-peak regions of the PSFs, such as the values for detectors 30-40 and 60-70 in the PSFs shown in Figure 24. This section addresses how the magnitude of collimator penetration scales with slit width and collimator thickness.

It is most straightforward to express the amount of transmission through the collimator in terms of an effective thickness, which is given in terms of the number of mean free paths. The effective thickness of the collimator can be inferred from the negative logarithm of the ratio of counts in the collimator penetration portion of the response to the corresponding value with no collimator present. This process isolates the effects of the collimator and eliminates the contributions of the solid angle and backscatter from the shielding and container that surrounds the detectors.

Before disentangling the contribution of backscattered neutrons, it is useful to be cognizant of their effects. Backscatter of neutrons from the shielding and container that surrounds the detectors not only increases the number of detected neutrons but also increases the amount of crosstalk between detectors. Since the performance of the imager is largely limited by inter-detector scattering, it is desirable to keep the amount of crosstalk to a minimum, and consequently, the amount of backscatter to a minimum. The 
ratio of neutrons backscattered from the outer imager shielding and reentering the detectors to the number leaving the detectors is shown in Figure 25 as a function of the thickness of the borated polyethylene shielding. With little shielding, the stainless steel container reflects approximately half of the neutrons back into the detectors. For borated polyethylene shielding thicknesses of $5 \mathrm{~cm}$ or greater, the amount of backscatter is reduced by about a factor of 2 . As a result, collimator configurations allowing at least $5 \mathrm{~cm}$ of borated polyethylene shielding on the outside of the detectors are preferred.

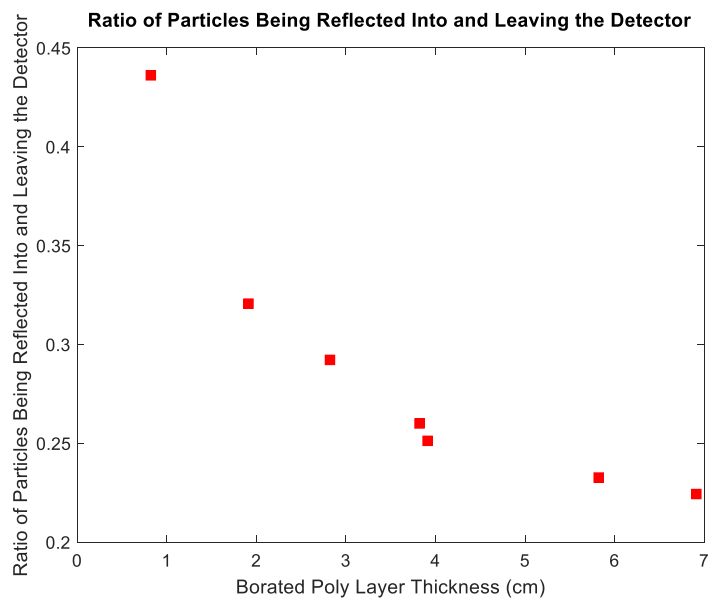

Figure 25. Ratio of neutron current leaving the detectors to the neutron current reflected back into the detectors.

Attenuation in the collimator is predominantly determined by the borated polyethylene portion. For a solid collimator, the thickness of the borated polyethylene collimator is the difference between its outer and inner diameters. However, for the collimators with slits, the average thickness of the collimator was taken to be the product of this linear distance and the closed fraction of the slits. In Figure 26, the effective collimator thickness is shown as a function of the average borated polyethylene thickness for six collimator configurations where the borated polyethylene portion is 35,40 , and $45 \mathrm{~cm}$ thick and the stainless steel is either 5 or $10 \mathrm{~cm}$ thick. Note that two trends are visible. One trend is the change in effective thickness due to changing the slit width (open fraction) of a collimator having given dimensions. This trend is visible in the slope of each collection of points of the same color. Another trend is the change in effective thickness due to changing the collimator dimensions. This trend is less obvious, but the blue and red points corresponding to thicker borated polyethylene collimators have increased effective thicknesses. These two trends have different slopes, indicating that the increase in dose rate with larger slits comes from two factors. One factor is the reduction of average collimator density. The other factor originates from radiation streaming down the slits to the detectors. These two trends will be discussed in more detail. 


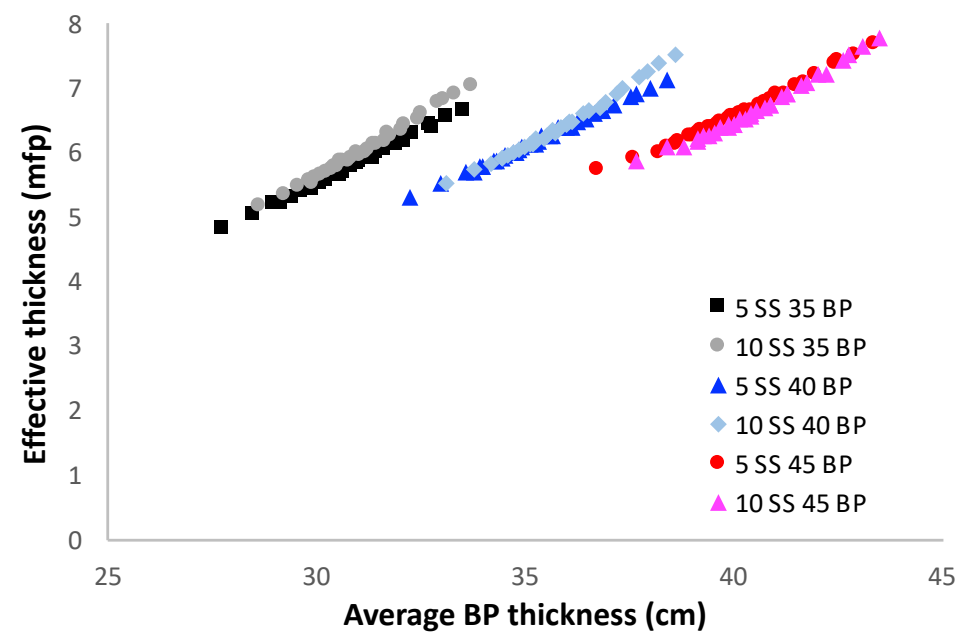

Figure 26. The effective thickness of the collimator inferred from the collimator penetration of the response as a function of the average thickness of borated polyethylene.

When considering the expected effective thickness of the collimator, an initial guess for a solid collimator would be that the thickness in mean free paths would equal the actual thickness times the macroscopic attenuation coefficient $\mu$, that is, $\mu t_{B P}$. Here, the attenuation coefficient is the inverse of the mean free path and equals the probability that a neutron will be removed per centimeter of material. This initial guess for the effective thickness can be corrected for the reduction in density due to the presence of slits by multiplying by $f_{\text {closedBP }}$, the fraction of the borated polyethylene portion of the collimator that consists of material rather than slit opening. Then, $\mu t_{B P} \rightarrow \mu t_{B P} f_{\text {closedBP. }}$. However, as neutrons pass through the collimator, lower-energy neutrons are preferentially captured. This preferential capturing means that the neutron spectrum hardens with additional borated polyethylene thickness, and the attenuation coefficient gets smaller. This neutron spectral hardening is manifest as a nonlinear relationship between the effective collimator thickness and the thickness of a solid collimator. In Figure 27, the effective thickness is shown as a function of the thickness of the (solid) borated polyethylene portion of the collimator. Over this limited range of thicknesses, the functional form of the attenuation coefficient can be estimated by a quadratic, $\mu \approx \mu_{0}\left(1-\Delta \mu t_{B P}\right)$.

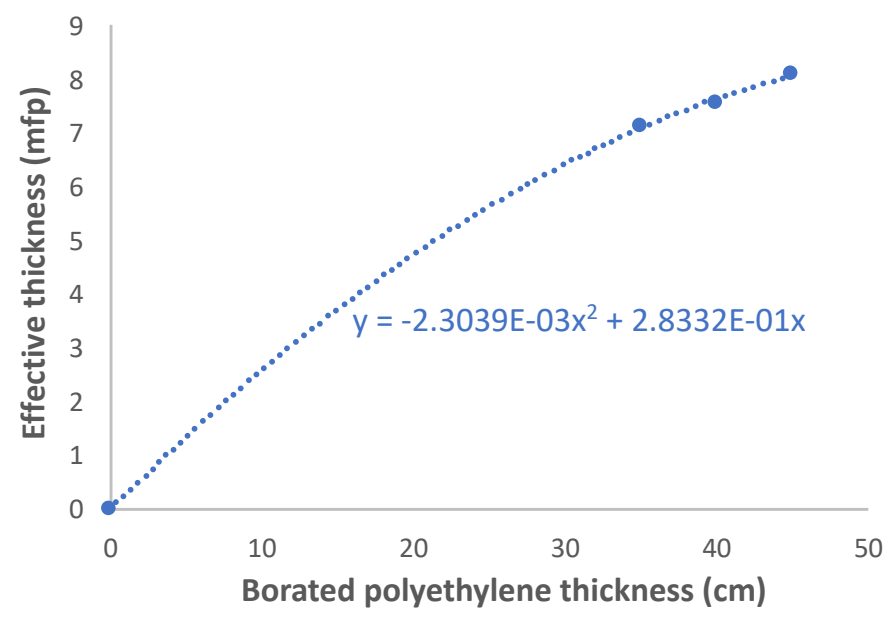

Figure 27. The effective thickness of a solid collimator in mean free paths as a function of the thickness of borated polyethylene. Note that hardening of the neutron spectrum with addition of polyethylene due to absorption of lower-energy neutrons is manifest in the nonlinearity. 
Using this thickness-dependent attenuation coefficient, the estimated thickness of the collimator can be written as

$$
\mu_{0} t_{B P} f_{\text {closedBP }}\left(1-\Delta \mu t_{B P} f_{\text {closedBP }}\right) .
$$

This equation takes into account the lower average density of the collimator and the change in attenuation coefficient with thickness in the collimator. However, this equation does not take into account streaming of radiation down the slit openings. The effect of radiation streaming can be seen by calculating the difference between the estimated thickness in Equation (3) and the effective thickness of the collimator. This difference is plotted in Figure 28 as a function of the missing thickness of borated polyethylene, $t_{B P}\left(1-f_{\text {closedBP }}\right)$, for collimator configurations consisting of $5 \mathrm{~cm}$ of stainless steel and 35,40 , and $45 \mathrm{~cm}$ of borated polyethylene.

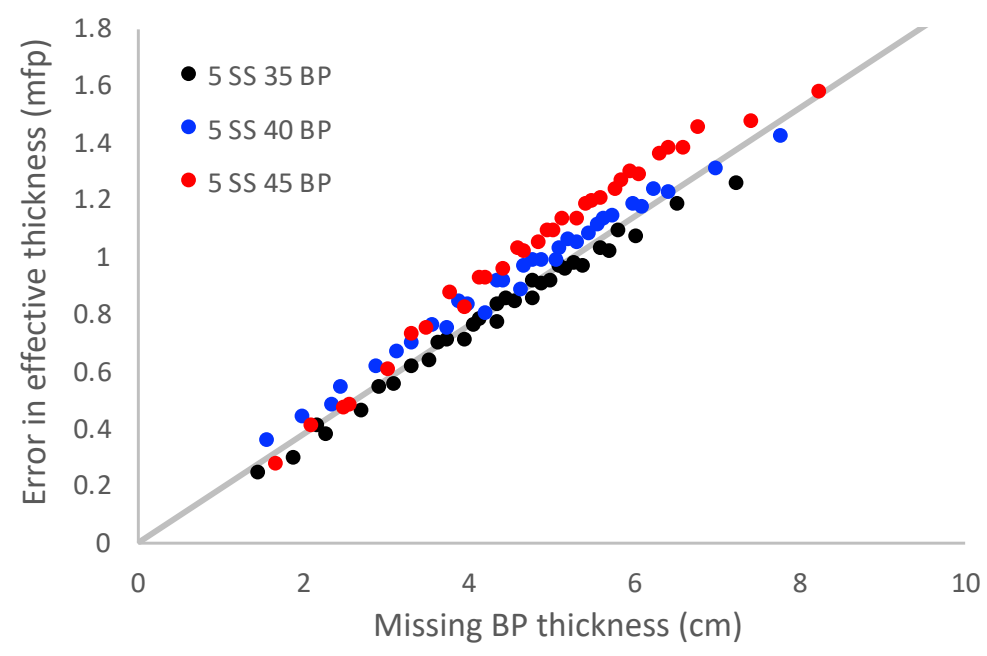

Figure 28. The difference between the calculated thickness (4) and the effective thickness of the collimator as a function of the thickness of borated polyethylene removed by the slits.

The close-to-linear dependence of the difference between the expected and effective thickness indicates that the effect of streaming down the slit openings can be represented as a correction that is linear in the open fraction of the slits. Then, the expected thickness of the collimator (in mean free paths) can be written as

$$
\mu_{0} t_{B P} f_{\text {closedBP }}\left(1-\Delta \mu t_{B P} f_{\text {closedBP }}\right)-C_{\text {slit }}\left(1-f_{\text {closedBP }}\right) t_{B P}
$$

Using this equation, and fitting for parameters $\mu_{0}, \Delta \mu$, and $C_{\text {slit }}$, the estimated thickness can be plotted as a function of the effective thickness, showing good general agreement. However, note that for different stainless steel thicknesses, different values of constants $\mu_{0}, \Delta \mu$, and $C_{s l i t}$, are required. This need can be seen in Figure 29, where the points corresponding to collimators having 5 or $10 \mathrm{~cm}$ of stainless steel fall on lines of slightly different slopes. However, making the constants $\mu_{0}, \Delta \mu$, and $C_{\text {slit }}$ a function of the stainless steel thickness did not improve the quality of the fit to all data, so these factors were not included in the discussion. 


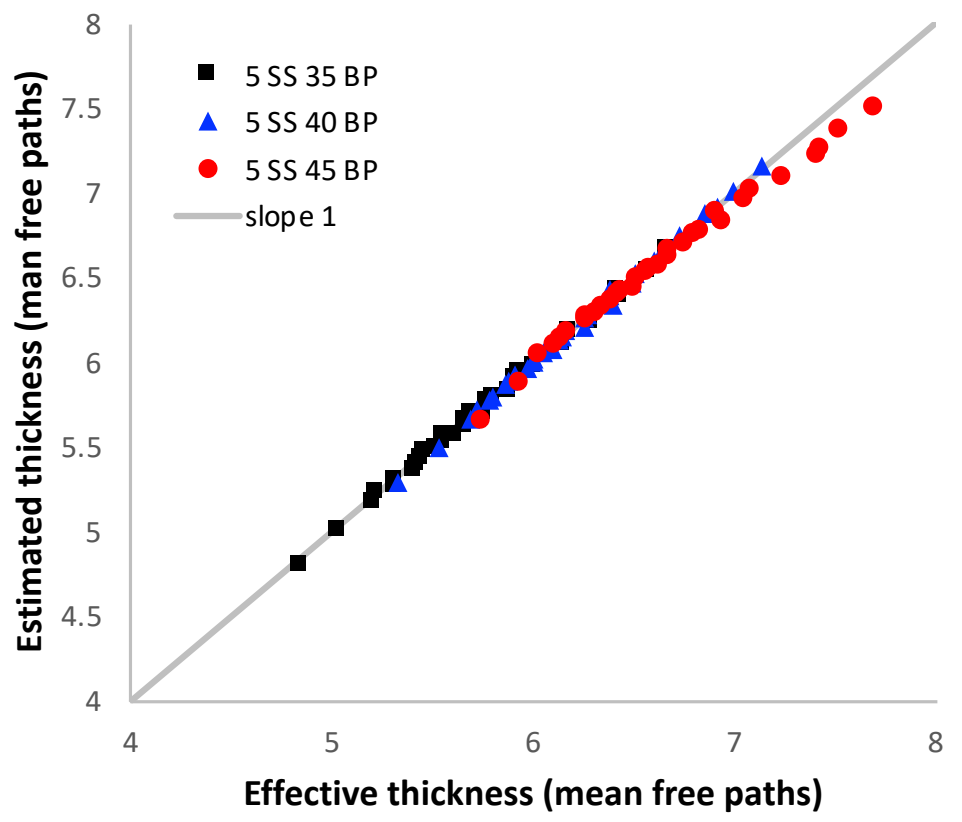

Figure 29. Estimated thickness, using Equation (4), as a function of the effective thickness of the collimator for configurations with $5 \mathrm{~cm}$ of stainless steel. For comparison, a line of slope=1 is shown gray.

\subsubsection{Signal to Noise Ratio}

In this section, an SNR is defined to identify desirable collimator configurations. To calculate the SNR, the implementation of an estimated collimator response is described. Then, the SNR is evaluated for 512 different collimator configurations to identify desirable configurations.

The best imager is the one that maximizes sensitivity to a missing fuel pin. In general, to best reconstruct an image of the fuel pins, the activity associated with each fuel pin needs to be attributed to the pin itself rather than the inter-pin space. In this context, we can define an SNR that captures the quality of data that feeds the image reconstruction. For an image reconstruction algorithm to attribute source intensity to the appropriate location, there needs to be a difference between the response to the fuel pin and the response to the inter-pin space. This difference should be as large as possible compared to statistical fluctuations. As a result, an appropriate definition of the "signal" is the magnitude of the difference between the calculated response of the imager to a single pin and to the same level of activity attributed to the "halo" surrounding that pin. An example of a pin and its corresponding halo is shown pictorially in Figure 30.
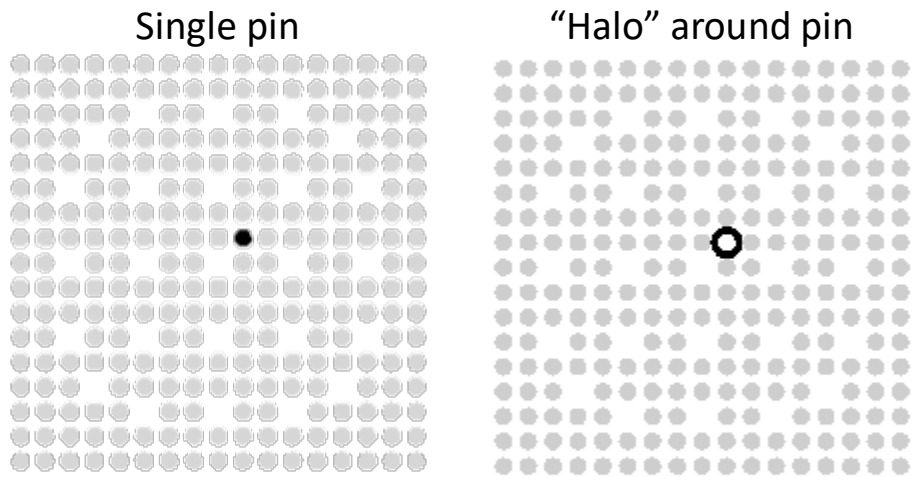

Figure 30. Image showing position of single pin and its associated halo. 
The magnitude of fluctuations of that difference, or the noise, is approximated by the square root of the neutron counts for the full assembly (in the region of interest for the particular fuel pin). The SNR can then be written to compare this signal to the fluctuations in counts from the full assembly; the result is given by Equation (5) below.

$$
S N R=\sum \frac{\mid \text { Response to pin }- \text { Response to halo } \mid}{\sqrt{\text { Response to assembly }}}
$$

To evaluate the SNR for each collimator configuration in the survey of configurations, the response of the collimator needed to be calculated on the millimeter length scale for the entire FOV for each projection angle. To reduce the computational burden associated with calculating the SNR for each configuration to a manageable level, an approximate imager response was developed. This approximate imager response was calculated over the entire FOV from a combination of the slit dimensions and the results of simulations of the response of a few points. Note that for calculating the SNR, it was not necessary to get all details of the estimated response correct, but it is important to get the relative magnitude between different configurations correct.

The approximate response was obtained from the constituent response components that were identified in the analysis of simulated responses, including the direct component of the response, the edge response, collimator penetration, and inter-detector scattering. The response to each slit was calculated over the entire FOV, and then inter-detector scattering was included via a weighted sum of individual slits. The response of an individual slit peaks at the sum of the direct and collimator penetration values and drops through the edge region to the value associated with collimator penetration.

The intensities of the direct and collimator penetration components can be easily inferred from a few simulated points. In particular, two points were simulated for each of the 512 collimator configurations. These points are shown on the left of Figure 23, and both points are aligned with slit 51 . The central (red) point was located $14.30 \mathrm{~cm}$ from the collimator opening and was used to determine collimator penetration. For this location, the magnitude of the collimator penetration was taken to be the average of detectors $25-30$ and 70-75 of the simulated response. For the purpose of the SNR calculation, the variation in collimator penetration due to differing local slit densities was ignored. For other points in the FOV, the value attributed to collimator penetration was scaled from this value by the ratio of the solid angle subtended by the detector at the position for which the response was being estimated to the solid angle subtended by the detector at the position for which the response was simulated.

The point closer to the slit opening (blue) was located $7.40 \mathrm{~cm}$ from the slit opening and was used to determine the direct component of the collimator response. The magnitude of the direct component was taken to be the difference between the peak value of the simulated response and the value of collimator penetration determined above. The value of the direct component for different distances from the detector was scaled from the ratio of solid angles to the simulated point.

Using the direct and collimator penetration values, the value of all points in the FOV due to a particular slit could be calculated provided the response in the edge region can also be estimated. This estimate was obtained by calculating the distance from the slit edge in units of projected slit width, per Figure 20. Then, for the purpose of the SNR calculations, the edge response was estimated by a linear drop in response from the collimator edge to 1.8 times the projected slit width.

An example estimated response for a single detector (detector 50) over the full FOV is shown for the baseline imager in Figure 31. On the left side of Figure 31, the position of detector 50 is indicated in red on the schematic diagram of the imager. In the middle plot, the response of detector 50 is shown in the absence of inter-detector scattering. Finally, the response to detector 50 including inter-detector 
scattering, where a neutron travels down a different slit but then scatters into detector 50 where it is detected, is shown on the right.
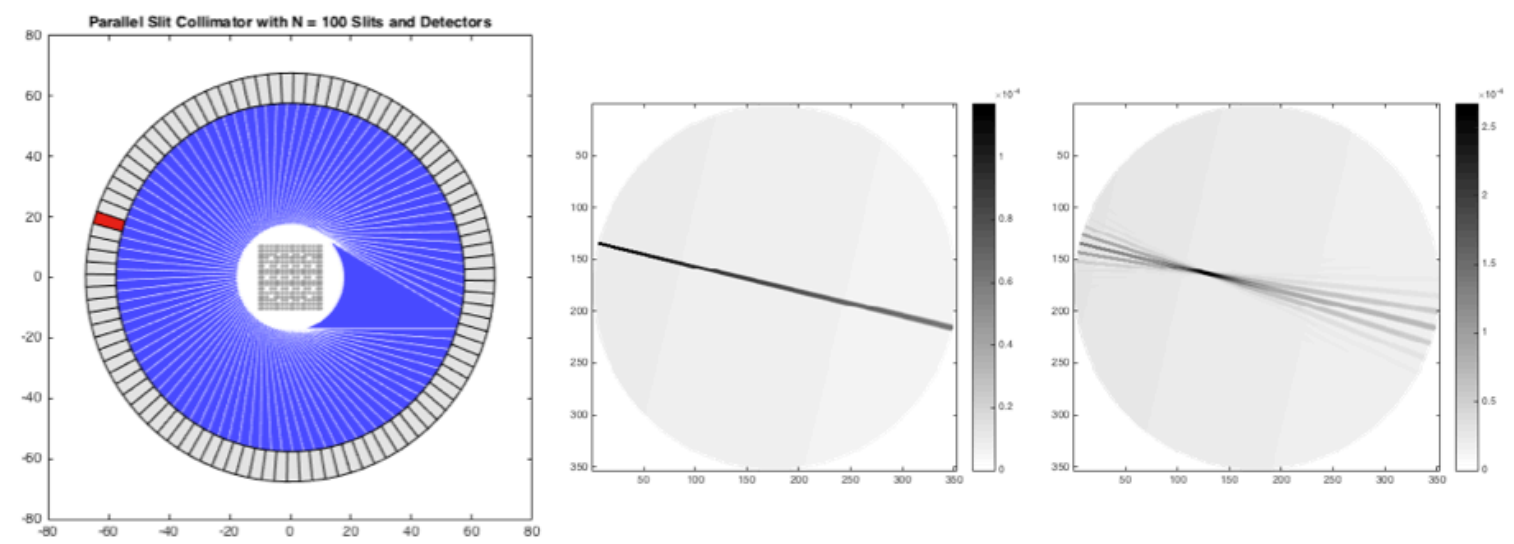

Figure 31. (Left) the baseline imager with detector 50 indicated in red, (center) the response to detector 50, and (right) the response to detector 50 including inter-detector scattering.

The estimated response was calculated for each of the 512 collimator configurations. Then, using the estimated response, the value of SNR was calculated for each configuration. Since the simulated response has units of counts per source neutron, for the SNR calculation, the detector response was scaled by 1 million emitted neutrons per fuel pin per projection. Then, for a fuel with $45 \mathrm{GWd} / \mathrm{MTU}$ burnup, this number of neutrons corresponds to a measurement time of approximately 3 seconds per projection or a total time of 5 minutes.

The SNR of all collimator configurations having thicknesses less than or equal to $45 \mathrm{~cm}$ are shown in Figure 32 as a function of the harmonic mean of the slit widths at the inner and outer collimator diameters, that is, the product of the slit widths at the inner and outer collimator diameters divided by the average of the widths. For slit widths less than about $0.4 \mathrm{~cm}$, the SNR for the various configurations scales approximately like the harmonic mean slit width, but for larger slit widths, the rate of increase slows. The harmonic mean is typically used for averaging rates, so in some sense the collimator can be thought of as passing a certain amount of signal rate in proportion to the aperture size through each end of the aperture so that the effective average of the two rates is proportional to the harmonic mean.

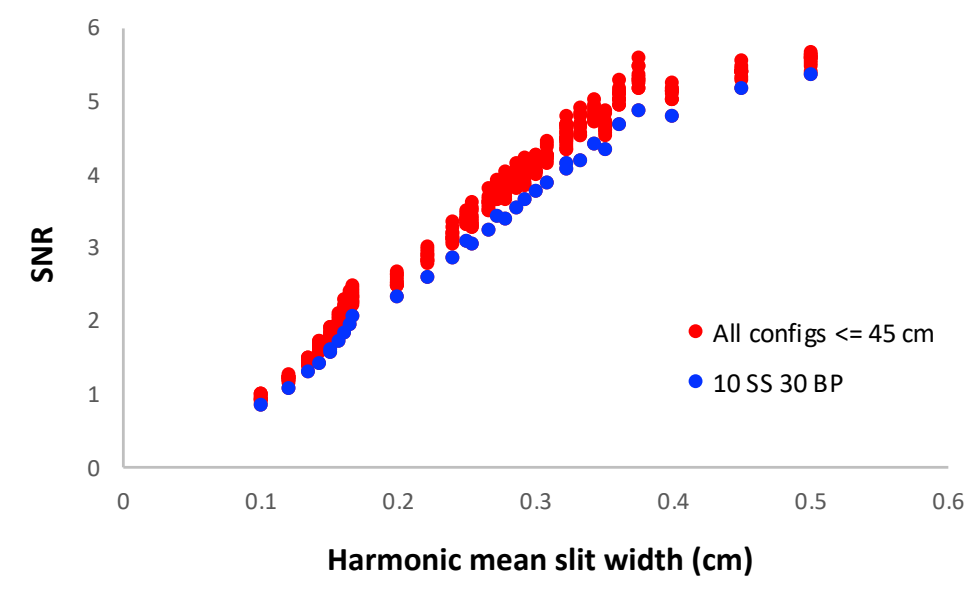

Figure 32. SNR for all 512 cases as a function of the slit harmonic mean. 
The values of SNR for all configurations with the same slit dimensions are approximately the same, but they increase slightly with increases in the fraction of collimator composed of borated polyethylene. Wider slit widths are favored in part because the collimator resolution is limited primarily by interdetector scattering, not slit width, so the increase in statistics outweighs a minimal loss in resolution.

The SNR can also be plotted as a function of the maximum detector dose rate of the configuration. This plot is shown in Figure 33 for all simulated configurations having a total thickness less than or equal to $45 \mathrm{~cm}$. As in Figure 32, the set of configurations with $10 \mathrm{~cm}$ of stainless steel and $30 \mathrm{~cm}$ borated polyethylene thicknesses are shown blue. However, although this configuration appears the worst in the plot of SNR as a function of slit width, it appears to be much more advantageous when plotting SNR as a function of gamma-ray dose rate.

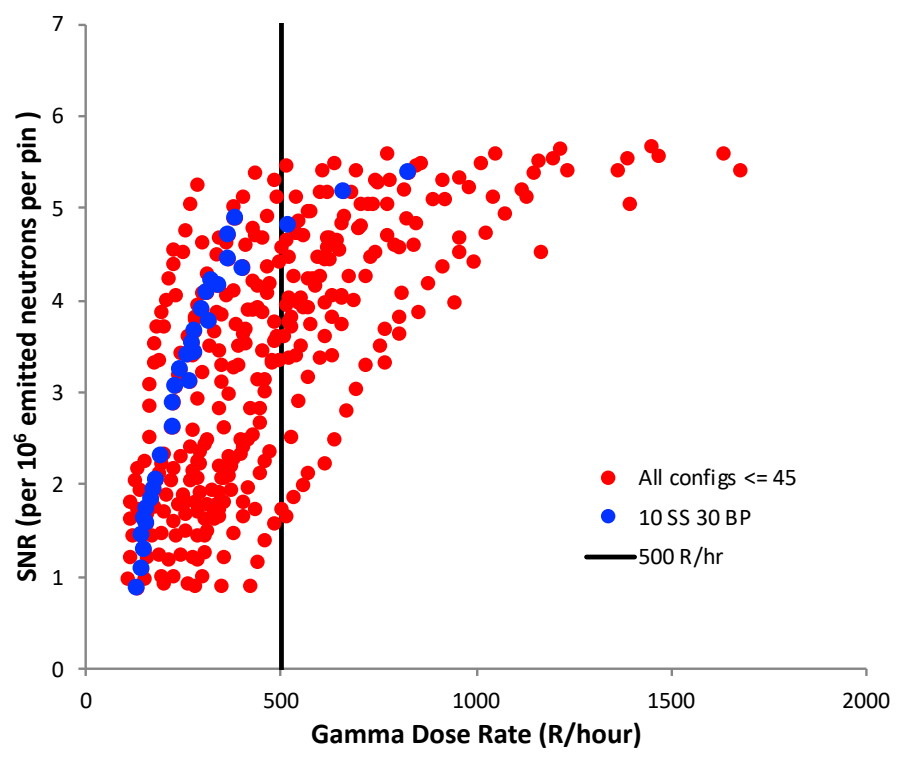

Figure 33. SNR as a function of the maximum gamma dose rate for each collimator geometry having a total collimator thickness less than or equal to $45 \mathrm{~cm}$. Collimator configurations with $10 \mathrm{~cm}$ stainless steel and $30 \mathrm{~cm}$ of borated polyethylene are shown blue.

There are a number of noteworthy observations about the data contained in the plot in Figure 33. First, the set of configurations associated with a particular stainless steel thickness and borated polyethylene thickness fall primarily along a trajectory, as illustrated by the blue points. Second, the main difference between configurations with different thicknesses is the gamma dose rate at the detectors, where the dose rate is lower for configurations having a larger diameter or a larger fraction of stainless steel. In contrast, the value of the SNR is similar for all configurations with the same slit width. Consequently, the primary benefit of thicker collimators is dose control, not SNR.

It also needs to be mentioned that the value of SNR was originally overestimated for slit widths greater than $3 \mathrm{~mm}$ at the collimator inner diameter. This overestimate resulted from using a source position that allowed, for the larger slit widths, neutrons to leak through the "edge" region of the slit response for neighboring slits, then scatter into the detector for which the response was being characterized. For the 3 , $3.5,4,4.5$, and $5 \mathrm{~mm}$ slit widths, this overestimation was a factor of 1.035, 1.084, 1.16, 1.29, and 1.50. The SNR values were correspondingly corrected by those factors. The SNR values for slit widths less than or equal to $3 \mathrm{~mm}$ are sufficiently accurate.

Since the most favorable design is the one that has the highest SNR at a dose rate that is tolerable (less than $500 \mathrm{R} \mathrm{h}^{-1}$ ), stainless steel improves collimator performance, not because it collimates neutrons 
effectively, but because it controls gamma dose rate and enables the use of wider, more efficient slits. Another path to dose control is the use of tapered slits. Tapered slits are also desirable because narrow slits in the steel region preserves the areal density of the collimator, whereas wider slits in the borated polyethylene region preserve efficiency.

Because the SNR performance of all the configurations of a particular slit width is similar, it is desirable to choose the most compact geometry where sufficient dose control can still be reasonably accomplished. In addition, geometries that can accommodate at least $5 \mathrm{~cm}$ of shielding on the outside of the detectors are desirable. As a result, configurations with $10 \mathrm{~cm}$ of stainless steel and $30 \mathrm{~cm}$ of borated polyethylene were preferred. Within this set, it was still the goal to achieve the best SNR while remaining within the maximum dose rate. With this goal in mind, additional tapered profiles for slits were considered. The performance of these additional tapered profiles was extrapolated from the set of tapered profiles that was simulated. In Figure 34, it can be seen that SNR and dose rate of tapered profiles having different slit widths at the collimator inner diameter fall roughly along lines. From a fit to these lines, the SNR and dose rate for tapered slits having a width of $3 \mathrm{~mm}$ at the narrow end and a range of slit widths at the wide end were calculated. In this instance, the $3 \mathrm{~mm}$ slit width was chosen because it corresponds to the notional spacing of slits in the parallel-slit collimator analog. In addition, the $3 \mathrm{~mm}$ width is sufficiently narrow to control gamma-ray dose.
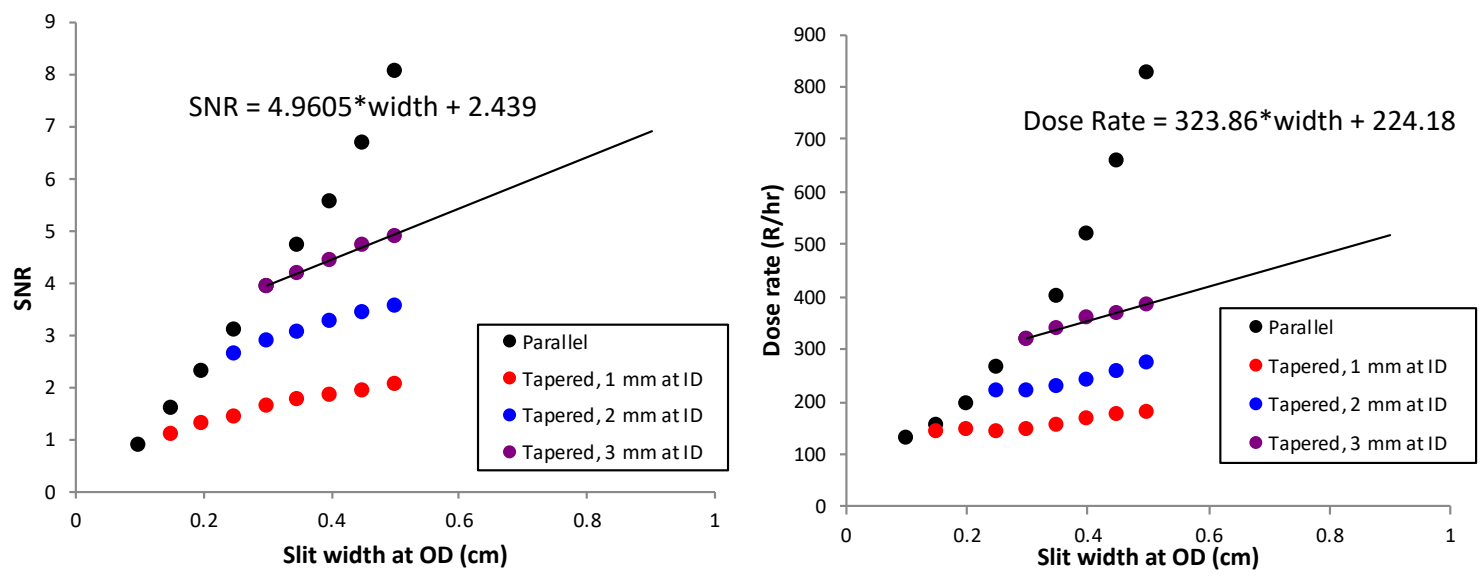

Figure 34. The SNR and dose rate as a function of slit width for parallel and tapered slits.

The estimated values of SNR and dose rate for tapered slits whose wide ends exceed $5 \mathrm{~mm}$ fall along the dashed line in Figure 35; the red point indicates the expected performance of slits with a width of $3 \mathrm{~mm}$ at the narrow end and $8 \mathrm{~mm}$ at the wide end. 


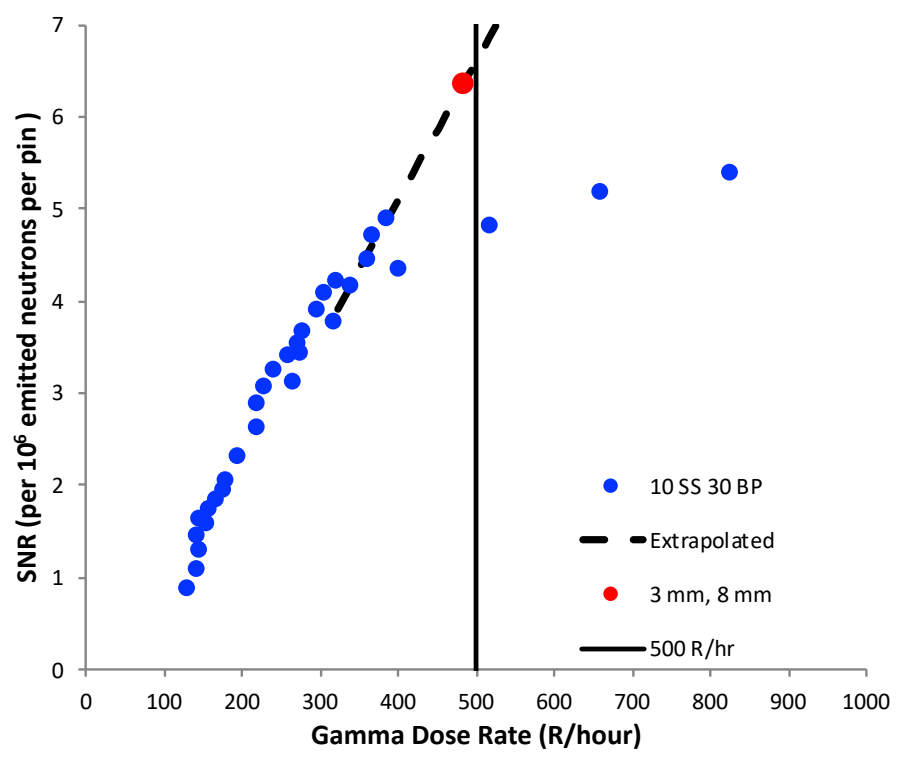

Figure 35. SNR as a function of the maximum gamma dose rate for collimator configurations consisting of $10 \mathrm{~cm}$ stainless steel and $30 \mathrm{~cm}$ of borated polyethylene. Tapered slits with a $3 \mathrm{~mm}$ width at the narrow end fall along the dashed line; the red point indicates an $8 \mathrm{~mm}$ width at the wide end.

This configuration gets close to the maximum value of SNR with a compact overall size. However, it is worth noting that the best collimator configuration for older, lower dose-rate fuels would likely be different. In particular, the lessened need for dose control would likely indicate wider, more efficient slits and perhaps less stainless steel.

\section{SUMMARY AND CONCLUSIONS}

Recently, ORNL devised the parallel-slit ring collimator concept. This novel collimator format is intended to enable a combination of efficiency, resolution, and radiation resistance that makes neutron imaging of spent fuel practical. The performance of the collimator is determined by the width of collimator slits and the thickness and materials used in its construction. In the present work, a simulation study was performed to identify configurations of this imager concept that minimize the time to identify individual fuel pins. In particular, collimator designs were considered that consist of an inner steel portion for structural integrity and dose control and an outer portion of borated polyethylene for modulation of neutrons.

For evaluating different collimator configurations, an SNR was defined that depends on the ability of the collimator to attribute activity to the fuel pins rather than the inter-pin gap. Then, desirable configurations were identified as those having a high SNR while maintaining a tolerable gamma-ray dose rate in the detectors. The tolerable dose rate in the detectors is limited to about $500 \mathrm{R} / \mathrm{h}$ by the low-probability, large amplitude interactions of single gamma rays in the boron-straw neutron detectors.

Simulations indicate that the collimator resolution is effectively limited by inter-detector scattering, not slit width. As a result, designs with more steel are preferred because, while steel poorly modulates neutrons, it effectively controls gamma dose so that wider, more efficient slits can be used. Simulations also find that the performance of collimator configurations varies only weakly with the collimator thickness. As a result, compact designs are preferred. Tapered slits offer a way to maximize performance from a compact design because they provide a desirable combination of dose control and efficiency; they are narrow in the steel region to preserve the areal density of the collimator and wider in the borated polyethylene region to preserve the efficiency of the collimator. Combining these preferences, a 
collimator composed of a $10 \mathrm{~cm}$ stainless steel section and a $30 \mathrm{~cm}$ borated polyethylene section with tapered slits with a width of $3 \mathrm{~mm}$ at the inner collimator radius and a width of $8 \mathrm{~mm}$ at the outer collimator radius produces a near-optimal value of SNR with a compact overall size.

As a final note, the "best" design is dose dependent. For the purposes of this simulation study, dose rates were calculated for a fuel having relatively high exposure (40 GWd/MTU) and a short cooling time (1 year). The high dose rate from this fuel favors designs having more steel and narrower slits. For older fuels with much smaller dose rates, designs having wider slits and less steel would be favored.

\section{ACKNOWLEDGEMENTS}

This work is supported by the support of the US Department of Energy, Office of Defense Nuclear Nonproliferation Research and Development in the National Nuclear Security Administration (NA-22). This research was also performed under appointment to the Nuclear Nonproliferation International Safeguards Fellowship Program sponsored by the National Nuclear Security Administration's Office of International Nuclear Safeguards (NA-241).

\section{REFERENCES}

[1] P. A. Hausladen, M. A. Blackston, and R. J. Newby. "Demonstration of Emitted-neutron Computed Tomography to Quantify Nuclear Materials.” Report no. ORNL/TM 357, Oak Ridge National Laboratory, Oak Ridge, TN, 2011.

[2] Y. Ham et al. "Partial Defect Verification of Spent Fuel Assemblies by PDET: Principle and Field Testing in Interim Spent Fuel Storage Facility (CLAB) in Sweden," Advancements in Nuclear Instrumentation Measurement Methods and their Applications (ANIMMA), 2015 4th International Conference, IEEE, 2015.

[3] L. Hildingsson and C, Andersson. "Safeguards Aspects Regarding a Geological Repository in Sweden," IAEA Symposium on International Safeguards Vienna, 2014.

[4] International Atomic Energy Agency, Department of Safeguards, Model Integrated Safeguards Approach for a Geological Repository, Report no. 03-SG-PR-1306,, International Atomic Energy Agency, Vienna, Austria, February 10, 2011.

[5] I. C. Gauld et al. "In-field Performance Testing of the Fork Detector for Quantitative Spent Fuel Verification," in ESARDA Symposium 2015, Manchester, UK, 2015.

[6] A. Lebrun and S. Zykov. "Status of NDA Techniques in use for IAEA Verification of Light Water Reactor Spent Fuel," Proceedings of 55th INMM Annual Meeting, 2014.

[7] “Pressurized Water Reactor (PWR) Systems,” Reactor Concepts Manual, USNRC Technical Training Center.

[8] T. Honkamaa, et al.,. “A Prototype for Passive Gamma Emission Tomography,” International Atomic Energy Agency Safeguards Symposium, 2014.

[9] International Atomic Energy Agency. "Safeguards Techniques and Equipment: 2011 Edition," International Atomic Energy Agency Verification Series No. 1 (Rev. 2), Vienna, 2011.

[10] S. P. Cerne, O. W. Hermann, R. M. Westfall, "Reactivity and Isotopic Composition of Spent PWR Fuel as a Function of Initial Enrichment, Burnup, and Cooling Time," Report no. ORNL/CSD/TM244, Oak Ridge National Laboratory, Oak Ridge, TN (1987).

[11] J. E. Fast et al. Spent Nuclear Fuel Measurements. Report no. PNNL-23561. Pacific Northwest National Laboratory, Richland, WA, 2014. 
[12] “Castor V/52." GNS Portfolio, GNS, www.gns.de/language=en/21553/castor-v-52.

[13] S. R. Greene, J. S. Medford, and S. A. Macy, "Storage and Transport Cask Data for Used Commercial Nuclear Fuel, 2013 U.S. Edition," Advanced Technology Insights LLC, Report no. ATI-TR-13047, August 2013, p. 34.

[14] F. G. Wouterlood. (2012). "Multiphoton Microscopy Advances Toward Super Resolution.” In Cellular Imaging Techniques for Neuroscience and Beyond (pp. 123-125). Waltham, MA: Academic Press. 